\title{
OPEN Sulf2a controls Shh-dependent neural fate specification in the developing spinal cord
}

\author{
Cathy Danesin $\bowtie$, Romain Darche-Gabinaud, Nathalie Escalas, Vanessa Bouguetoch, \\ Philippe Cochard, Amir AI Oustah, David Ohayon, Bruno Glise \& Cathy Soula
}

Sulf2a belongs to the Sulf family of extracellular sulfatases which selectively remove 6-0-sulfate groups from heparan sulfates, a critical regulation level for their role in modulating the activity of signalling molecules. Data presented here define Sulf2a as a novel player in the control of Sonic Hedgehog (Shh)-mediated cell type specification during spinal cord development. We show that Sulf2a depletion in zebrafish results in overproduction of V3 interneurons at the expense of motor neurons and also impedes generation of oligodendrocyte precursor cells (OPCs), three cell types that depend on Shh for their generation. We provide evidence that Sulf2a, expressed in a spatially restricted progenitor domain, acts by maintaining the correct patterning and specification of ventral progenitors. More specifically, Sulf2a prevents Olig2 progenitors to activate high-threshold Shh response and, thereby, to adopt a V3 interneuron fate, thus ensuring proper production of motor neurons and OPCs. We propose a model in which Sulf2a reduces Shh signalling levels in responding cells by decreasing their sensitivity to the morphogen factor. More generally, our work, revealing that, in contrast to its paralog Sulf1, Sulf2a regulates neural fate specification in Shh target cells, provides direct evidence of non-redundant functions of Sulfs in the developing spinal cord.

Sulf protein family, comprising Sulf1 and Sulf2, are heparan sulfate (HS) proteoglycan-editing enzymes that regulate a large number of signalling pathways ${ }^{1,2}$. These extracellular enzymes have been shown to present similar enzymatic activity, remodelling the 6-O-sulfation state of HS chains on the cell surface ${ }^{3-5}$. Thereby, Sulfs modulate HS binding to signalling molecules which leads to either promoting or inhibiting their signalling activities ${ }^{1,2}$. Sulf1 is the first discovered Sulf that was identified as a developmentally regulated protein, modulating Wnt signalling during somitogenesis in quail ${ }^{6}$. Since then, Sulf1 has been shown to control the signalling activity of other morphogen factors, including Sonic Hedgehog (Shh $)^{7-11}$. In particular, Sulf1 behaves as a positive modulator of Shh signalling during spinal cord development, where it appears central in governing ventral neuron and oligodendrocyte specification ${ }^{7-9}$. How Sulf1 controls Shh signalling in the embryonic spinal cord has been partially elucidated ${ }^{12}$. In the ventral spinal cord, Shh signalling regulates generation of different types of neurons and glial cells which differentiate from distinct neural progenitors arrayed in domains along the dorso-ventral axis of the progenitor zone. Formation of these domains occurs early during neural tube patterning. Shh, emanating first from the notochord and later from the medial floor plate, spreads dorsally and induces expression of specific sets of transcription factors that trigger the formation of progenitor domains, each dedicated to generate a specific subtype of ventral neurons ${ }^{13,14}$. These domains emerge in a progressive manner and their order of appearance corresponds with their requirement for increasing concentrations and durations of Shh signalling ${ }^{15,16}$. This is illustrated by the sequential activation of Olig2 and Nkx2.2, two transcription factors expressed in the ventral-most progenitor domains. Nkx2.2, expressed in progenitors of the $\mathrm{p} 3$ domain and required for $\mathrm{V} 3$ interneuron specification, is located ventrally to progenitors of the pMN domain, which express Olig2 and generate somatic motor neurons $(\mathrm{MNs})^{13}$. During patterning establishment, and in response to Shh, ventral neural progenitors first activate expression of Olig2. Afterwards, in response to a temporal increase in Shh signalling activity, the ventral-most progenitors activate Nkx2.2, a high-threshold Shh responsive gene. $\mathrm{Nkx} 2.2$, in turn, represses Olig2, thus leading to formation of the $\mathrm{p} 3$ and $\mathrm{pMN}$ non-overlapping domains. This is precisely at this step that Sulf1 is intervening. The enzyme, which is up-regulated in medial floor plate cells just prior to Nkx2.2 up-regulation, enhances Shh signalling activity by stimulating production of active forms of Shh from its source cells ${ }^{7}$. Noticeably, Shh stimulatory function of Sulf1 is reused later, at a time when pMN cells stop generating MNs and change their fate to generate oligodendrocyte precursor cells (OPCs). This temporal cell 
fate change depends on the formation of a novel source of Shh, named the lateral floor plate, that forms within the Nkx2.2-expressing progenitors of the $\mathrm{p} 3$ domain ${ }^{12}$. Again, Sulf1 must be up-regulated in these Shh-secreting cells so that these cells provide higher Shh signal to neighbouring $\mathrm{pMN}$ cells which, in turn, activate expression of Nkx2.2 that no longer represses Olig2 at this stage ${ }^{17-20}$. In this way, Sulf1 contributes to induce formation of a new progenitor domain, named the $\mathrm{p}^{*}$ domain, populated by cells co-expressing Olig 2 and Nkx 2.2 fated to generate OPCs. Thus, in the developing spinal cord, the key role of Sulf1 is to change the inductive properties of Shh source cells to trigger high-threshold response to Shh.

Much less is known about the role of Sulf 2 in regulating morphogen signalling. Sulf1 and Sulf 2 have been proposed to display compensatory functions in many developmental processes ${ }^{3,4,21-23}$. However, whether Sulf1 and Sulf2 exert specific roles in the developing spinal cord remains to be addressed. In this tissue, in contrast to Sulf1 whose expression is restricted to medial and lateral floor plate cells, Sulf2 is expressed broadly in both floor plate cells and neural progenitors ${ }^{7-11,24-26}$. Comparison of Sulf1 and Sulf2 mouse knockout phenotypes led to the conclusion that the two enzymes act in a similar way at the level of Shh source cells to regulate Shhdependent patterning at the time of OPC specification ${ }^{11}$. However, supporting the view that Sulf2 has a unique and additional function, conditional depletion of Sulf2 specifically in neural progenitors expressing Olig2 but not in Shh source cells is sufficient to impair gliogenesis in mouse $\mathrm{s}^{26}$. Whether this function is linked to regulation of the Shh signal remains an open question.

In zebrafish, three Sulf members have been identified: Sulf1 and two Sulf2 paralogs, named Sulf2a and Sulf $2 b^{27}$. Here, we report that Sulf2a is the unique Sulf member whose expression is restricted to neural progenitors, then offering a simple context to study Sulf function specifically in Shh-responding cells of the developing spinal cord. We show that Sulf2a is required for proper generation of ventral neuronal and oligodendroglial cell subtypes and provide evidence that Sulf2a plays a key role in controlling neural progenitor identity. Overall, our data support a model whereby Sulf2a, by reducing the sensitivity of target cells to Shh, promotes low-threshold response to the morphogen over spinal cord development.

\section{Results}

Sulf $2 a$ expression is restricted to neural progenitors of the developing spinal cord. As a first step to investigate the function of Sulf2 proteins in zebrafish, we analysed expression patterns of sulf $2 a$ and sulf $2 b$. We first focused on two development stages: $24 \mathrm{hpf}$, while ventral neural pattern is established and neuronal production is ongoing and $36 \mathrm{hpf}$, corresponding to the time of $\mathrm{p}^{*}$ domain formation and onset of OPC production in the ventral spinal cord. As previously reported ${ }^{27}$, we found that, at neurogenic stage (24 hpf), sulf $2 b$ is expressed in medial floor plate cells but also in adjacent neural progenitors of the p3 domain (Fig. 1a, b). At the onset of gliogenesis ( $36 \mathrm{hpf}$ ), sulf $2 b$ signal, although weaker, was still detected in the medial floor plate and in adjacent cells which form to the lateral floor plate at that stage (Fig. 1c, d). Thus, as previously reported for Sulf $1^{7}$, sulf $2 b$ is expressed in Shh-producing cells of the developing spinal cord. Interestingly, we found a very different pattern of expression for sulf $2 a$ whose mRNA was detected in a broad domain of the ventral spinal cord at both neurogenic and gliogenic stages (Fig. 1e-h). Noticeably, sulf $2 a$ mRNA was undetectable either in medial floor plate or in lateral floor plate cells but was found expressed in neural progenitors located at distance from Shh-producing cells (Fig. 1f, h). Double detection of sulf $2 a$ and shh mRNAs further confirmed that sulf2a and shh domains of expression do not overlap neither at neurogenic ( $24 \mathrm{hpf}$ ) nor gliogenic ( $36 \mathrm{hpf}$ ) stages (Fig. 1k, 1). These data therefore revealed a unique property of sulf $2 a$ being exclusively expressed in neural progenitors.

We next investigated sulf $2 a$ expression at earlier and later stages of development. Ruling out the possibility that sulf $2 a$ plays a role in establishment of progenitor domains, we found that sulf $2 a$ is not yet expressed at 16 hpf (Fig. 1i), while neural tube patterning has yet been established and neuronal production has already been initiated ${ }^{7,28}$. Much later, at the end of embryogenesis ( $72 \mathrm{hpf}$ ), sulf $2 a$ expression was maintained in neural progenitors and was still excluded from Shh-producing cells (Fig. 1j), thus indicating that sulf2a remains expressed in neural progenitors after the onset of neuronal production and all along gliogenesis.

Together, these results, showing that sulf $2 a$ is the only sulf gene activated specifically in neural progenitors open the possibility that this Sulf member plays a specific role in regulating signal reception at the surface of spinal progenitors as they generate neurons and glial cells.

Sulf2a controls generation of both V3 interneurons and OPCs. Our data showing that sulf $2 a$ is activated after initiation of neuronal production and persists along gliogenesis, prompted us to examine its function in regulating generation of V3 interneurons and OPCs produced by ventral neural progenitors ${ }^{29,30}$. To address the role of Sulf2a, we first used a morpholino (MO) knockdown approach, using a translation (sul$f 2 a \mathrm{MO}^{\mathrm{ATG}}$ ) and a splice (sulf2aMO ${ }^{\text {splice }}$ ) blocking morpholino. We performed RT-PCR which highlighted the presence of unspliced forms of the sulf $2 a$ transcript in sulf $2 a \mathrm{MO}^{\text {splice }}$-injected embryos, showing knockdown efficiency in these experiments (Supplementary Fig. 1a, b). As similar results were obtained using either MO in all experiments, they are referred to as sulf $2 a \mathrm{MO}$ in the text. We first assessed V3 interneuron generation by detecting sim $1 a$ expression as a specific marker of these neurons originating from the $\mathrm{p} 3$ domain ${ }^{29,31}$. At 48 hpf, as neuronal production has been completed, we found that the number of $\operatorname{sim} 1 a+$ cells was significantly higher in sulf2aMO-injected embryos compared to the control MO (ctrlMO)-injected ones, with more than a $20 \%$ increase in the number of V3 interneurons in sulf $2 a$ morphants (Fig. 2a-c, f). To confirm these observations, we generated a sulf2a mutant zebrafish line using the CRISPR/Cas9 system. We targeted the hydrophilic domain (HD) region of Sulf2a, known to be required for enzymatic activity and substrate recognition ${ }^{3,32-34}$. We obtained an indel mutation in the coding region which leads to a premature translation termination and a predicted protein missing functional HD domain (Supplementary Fig. 2a). Validating that this mutation is indeed a loss-of-function allele, sulf $2 a$ mRNA was found nearly undetectable in embryos carrying homozygous 


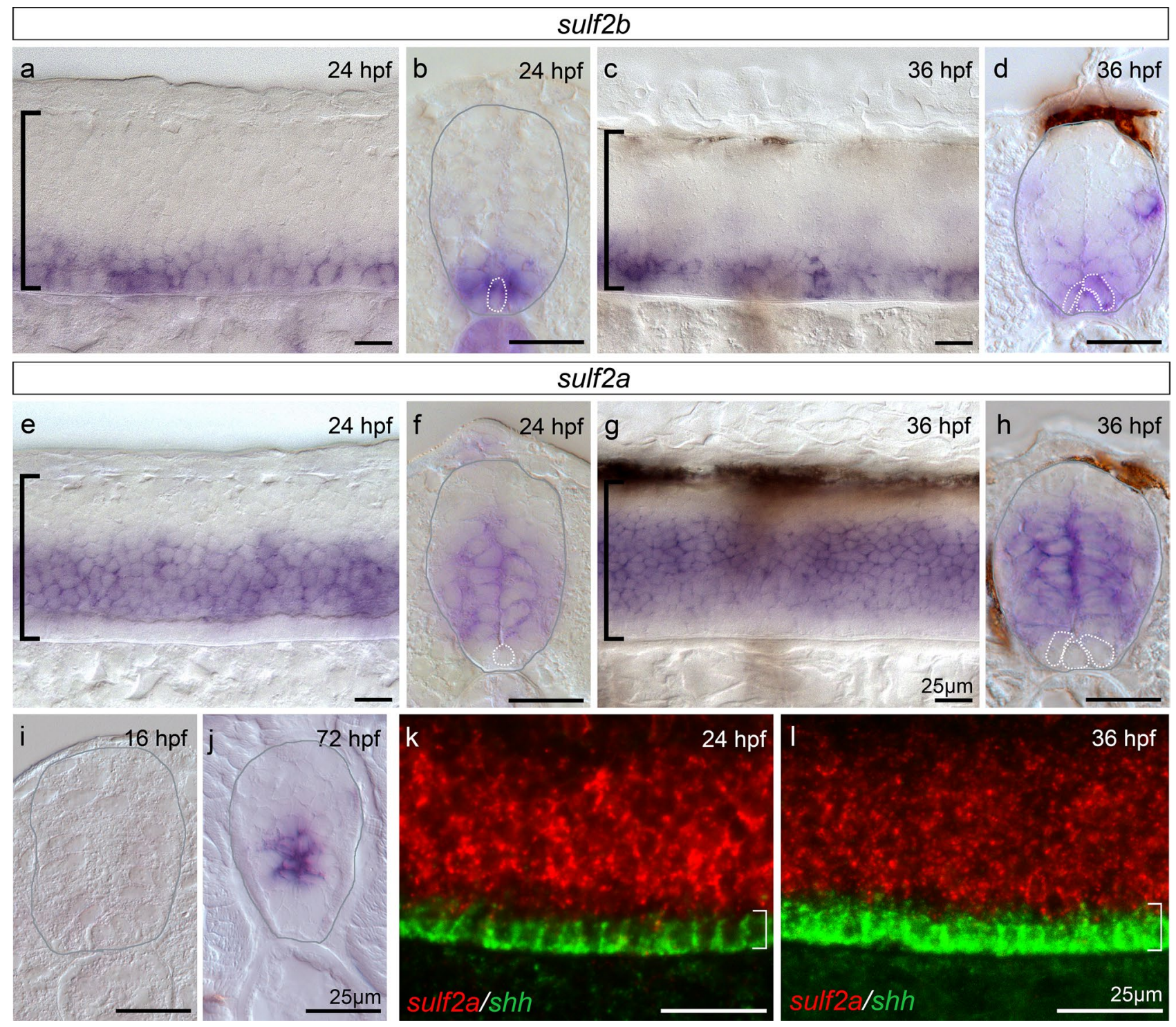

Figure 1. Sulf $2 b$ is expressed within Shh-producing cells whereas Sulf $2 a$ is restricted to neural progenitors. Here and in all subsequent panels, side views of embryos (a,c,e,g,k,l) are at the level of the trunk spinal cord, anterior to the left and dorsal to the top, and spinal cord transverse sections $(\mathbf{b}, \mathbf{d}, \mathbf{f}, \mathbf{h}, \mathbf{i}, \mathbf{j})$ are shown dorsal to the top. Developmental stages are indicated in each panel. $(\mathbf{a}-\mathbf{j})$ Detection of $s u l f 2 b(\mathrm{a}-\mathrm{d})$ and $s u l f 2 a(\mathrm{e}-\mathrm{j}) \mathrm{mRNAs}$ by whole-mount in situ hybridisation. Images are representative examples of at least five embryos. Grey outlines $(\mathbf{b}, \mathbf{d}, \mathbf{f}, \mathbf{h}, \mathbf{i}, \mathbf{j})$ label the spinal cord edge. Brackets in $\mathbf{a}, \mathbf{c}, \mathbf{e}, \mathbf{g}$ indicate the position of the spinal cord. Dotted lines in b,d,f,h) delineate Shh-producing cells, i.e. the medial floor plate at $24 \mathrm{hpf}$ and the medial floor plate + lateral floor plat at $36 \mathrm{hpf}$. Note that sulf2a mRNA is detected in neural progenitors but not in medial and lateral floor plate cells. (k-1) Double detection of sulf $2 a$ (red) and shh (green) mRNAs by fluorescent in situ hybridisation, showing that sulf $2 a$ expression does not overlap the shh-expressing domain (white brackets).

sulf $2 a$ mutation (sulf2a-/-) (Supplementary Fig. 2b). Adult sulf2a-/-individuals showed no overt phenotype, are viable, fertile, and produce offsprings that show no obvious morphological defects. Confirming the requirement of Sulf2a to restrict V3 interneuron production, we found that the number of sim 1a + neurons increased by $25 \%$ in sulf2a-/- embryos compared to wild-type siblings (Fig. 2d-f). We conclude from these data that Sulf2a plays a role in limiting production of $\mathrm{V} 3$ interneurons.

Sulf2a expression being maintained at $36 \mathrm{hpf}$, the time of OPC specification ${ }^{30}$, we next asked whether Sulf2a might also play a role in the control of OPC generation. To identify OPCs, we used either $\operatorname{Tg}($ olig2:GFP) or $\operatorname{Tg}$ (olig2:DsRed2) transgenic lines in which fluorescently-labelled OPCs can be identified by their morphology and position ${ }^{35,36}$. Due to expression of olig2 in progenitors of the pMN domain as well as perdurance of GFP or DsRed 2 in newly generated $\mathrm{MNs}^{35-37}$, these reporter lines however do not allow distinguishing OPCs from other cell types in the ventral spinal cord. We bypassed this problem by performing immunodetection of Sox 10, the earliest specific marker of oligodendroglial cells ${ }^{37}$. At $48 \mathrm{hpf}$, when OPC generation has just began, we found olig2:GFP + or olig2:DsRed2 + cells co-expressing Sox10 in the ventral spinal cord of both ctrlMO-injected and wild-type embryos (Fig. 3a, d). At identical stage, the number of OPCs was significantly reduced either in 


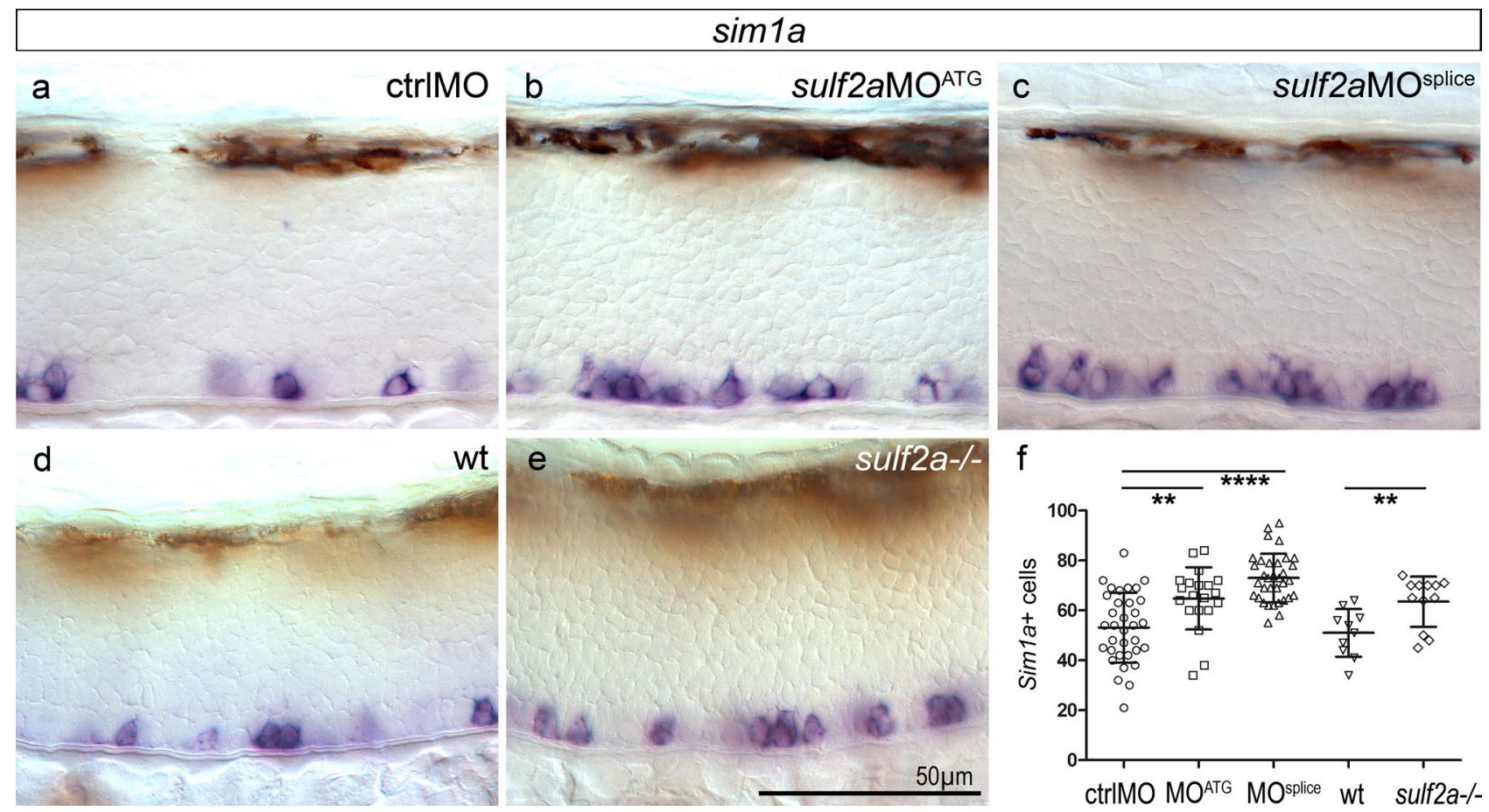

Figure 2. V3 interneuron production is increased upon Sulf $2 a$ depletion. Side views of $48 \mathrm{hpf}$ embryos. (a-f) Detection (a-e) and quantification ( $\mathrm{f}$ ) of sim1a-expressing neurons in embryos injected with ctrlMO $(\mathbf{a}, \mathrm{n}=34)$, sulf2aMO ${ }^{\text {ATG }}(\mathbf{b}, \mathrm{n}=20)$ or sulf $2 a \mathrm{MO}^{\text {splice }}(\mathbf{c}, \mathrm{n}=34)$ and in wild-type $(\mathbf{d}, \mathrm{n}=10)$ or sulf $2 a-/-(\mathbf{e}, \mathrm{n}=12)$ embryos from two independent experiments in each case. Datasets were compared with Mann Whitney's test (twotailed). Data are presented as mean number of cells per embryo \pm s.d $\left({ }^{* *} \mathrm{p}<0.01,{ }^{* * * *} \mathrm{p}<0.0001\right)$.
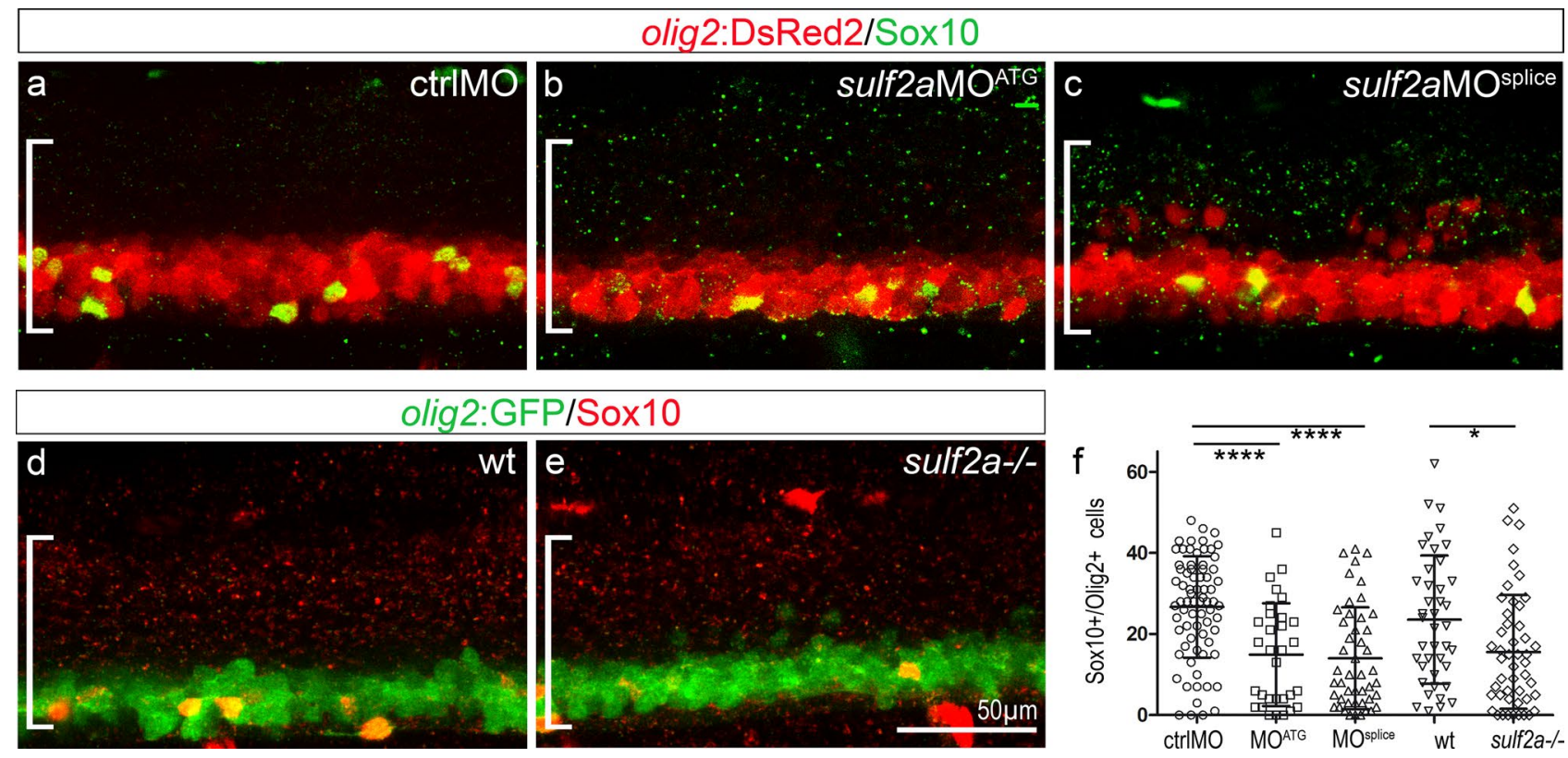

Figure 3. Sulf2a depletion impairs OPC development. Side views of $48 \mathrm{hpf}$ embryos. (a-f) Detection (a-e) and quantification (f) of OPCs by immunodetection of Sox10 (green in a-c, red in d,e) in Tg(olig2:DsRed) embryos (red in $\mathrm{a}-\mathrm{c})$ injected with ctrlMO $(\mathrm{a}, \mathrm{n}=73)$, sulf2aMO $\mathrm{aTG}^{\mathrm{AT}}(\mathrm{b}, \mathrm{n}=31)$ or sulf $2 a \mathrm{MO}^{\text {splice }}(\mathrm{c}, \mathrm{n}=47)$ and wild-type $(\mathrm{d}, \mathrm{n}=10)$ or sulf2a-/- $(\mathrm{e}, \mathrm{n}=48) \mathrm{Tg}($ olig2:GFP) embryos (green in d,e) from 9 and 6 independent experiments, respectively. Datasets were compared with Mann Whitney's test (two-tailed). Data are presented as mean number of cells per embryo \pm s.d $\left({ }^{*} \mathrm{p}<0.05,{ }^{* * * *} \mathrm{p}<0.0001\right)$. 
sulf $2 a \mathrm{MO}$-injected or in sulf $2 a-/-$ embryos, with a decrease of more than $45 \%$ and $33 \%$ compared to controls (Fig. 3b, c, e, f). In order to assess the specific involvement of sulf $2 a$ in OPC development, we performed rescue experiments and found that co-injection of sulf $2 a \mathrm{MO}^{\text {splice }}$ together with sulf $2 a$ mRNA, which thus does not carry the MO targeted nucleotide sequence, restored the OPC number to control level (Supplementary Fig. 1c-f). Thus, our data evidenced that Sulf2a activity is also required for proper generation of OPCs but, instead of limiting the number of cells as observed for V3 interneurons, the enzyme contributes to promote OPC production.

Together, these results, showing that Sulf2a limits production of V3 interneurons during neurogenesis but promotes generation of OPCs at initiation of gliogenesis, reveal specific and differential functions of Sulf2a in controlling generation of ventral neural cell subtypes.

Sulf2a controls the identity of ventral neural progenitors. We next focused on the question of how the same enzyme can fulfil differential regulatory functions to ensure production of a correct number of neural derivatives. Sulf2a being expressed in neural progenitors, we postulated that the enzyme might be involved in regulating progenitor patterning and subsequent cell diversification. We started analysing progenitor domain organisation at $24 \mathrm{hpf}$ while neurons are being produced. We focused on the $\mathrm{p} 3$ and $\mathrm{pMN}$ domains characterised by the expression of $\mathrm{Nkx} 2.2 \mathrm{a}$ and Olig2, respectively. Nkx2.2a expression was assessed detecting mEGFP in the $\operatorname{Tg}\left(n k \times 2.2 a\right.$ :mEGFP) line ${ }^{36}$, while Olig2 expression was monitored by detecting the olig2 mRNA using fluorescent RNA in situ hybridisation. Immunodetection of Sox2, a generic marker of neural progenitors ${ }^{38,39}$, was also performed to distinguish these cells from newly generated neurons. Analysis of ctrlMO-injected embryos revealed $n k x 2.2 a$ :mEGFP + progenitors organised in a row of one to two cells in the ventral progenitor zone and comparison with olig2 mRNA staining showed that olig2 expression defined the dorsally adjacent nonoverlapping progenitor domain (Fig. 4a-a”), thus reflecting ventral patterning establishment. Similar analysis performed in sulf $2 a \mathrm{MO}$-injected embryos showed that the $n k x 2.2 a$ :mEGFP + domain extended dorsally, covering rows of two to three cells, reflecting a more than $35 \%$ broadening of the $\mathrm{p} 3$ domain compared to controls (Fig. 4b, b", c, c", d). Thus, in agreement with the excess of V3 interneurons observed in Sulf2a-depleted embryos, $\mathrm{p} 3$ progenitor number is increased in these embryos. By contrast, olig2 expression was found markedly reduced in sulf $2 a \mathrm{MO}$-injected embryos (Fig. 4b' c"). Counting of olig2 + progenitors indicated a more than 59\% decrease in sulf $2 a$ morphants compared to control embryos (Fig. 4e). Accordingly, the number of MNs, assessed by immunostaining of Islet $1 / 2$ in $\operatorname{Tg}($ olig2:DsRed2) embryos, was reduced by $20-30 \%$ in $48 \mathrm{hpf}$ sulf $2 a$-depleted embryos (Supplementary Fig. 3). Together, these data indicated that Sulf2a is part of the regulatory process that controls dorsal boundary positioning of the $\mathrm{Nkx} 2.2 \mathrm{a} / \mathrm{p} 3$ domain and thus contributes to maintain the Olig2/ pMN domain over the period of neuronal production.

We next examined neural patterning at the onset of gliogenesis ( $36 \mathrm{hpf})$. As expected, we found that, in control embryos, the $n k x 2.2 a$ :mEGFP + progenitor domain was broader than that observed at the neurogenic period (compare Figs. 4 and 5) and partially overlapped the olig2-expressing domain (Fig. 5a", a"), reflecting formation of the $\mathrm{p}^{\star}$ domain at this stage. As observed at neurogenic period, sulf $2 a$ depletion caused dorsal expansion of the $n k x 2.2 a$ :mEGFP + domain, with a more than $25 \%$ increase in the number of $n k x 2.2 a: m E G F P+$ progenitors compared to controls (Fig. 5b, b", b"', c", c'”, d), thus indicating that the dorsal boundary position of the Nkx2.2aexpressing domain is also affected by Sulf2a depletion at the time of patterning rearrangement. Counting of olig 2 + progenitors in sulf $2 a$ morphants showed that their number decreased by $40-50 \%$, a reduction still significant but less pronounced than that observed at neurogenic stage (Fig. 5b', c', e). Noticeably and in agreement with the loss of repressive activity of $\mathrm{Nkx} 2.2 \mathrm{a}$ on olig2 expression at this stage, no significant difference was found in the number of progenitors co-expressing $n k x 2.2 a$ :mEGFP and olig2 between control and sulf $2 a$ morphant embryos (Fig. 5a"-c"', f). The logical corollary of this latter observation is that, upon sulf $2 a$ depletion, the observed reduction in the number of Olig2 progenitors should be due to a specific decrease in the number of progenitors expressing Olig2 but not Nkx2.2a. Accordingly, quantification of these cells showed a decrease by more than $60 \%$ in sulf $2 a$-depleted embryos (Fig. 5a" $-c$ ', g). Thus, at gliogenic stage, Sulf2a depletion causes dorsal expansion of the $\mathrm{Nkx} 2.2 \mathrm{a}$-expressing domain, does not affect generation of the $\mathrm{p}^{*}$ domain but leads to a drastic loss of the dorsally located progenitors that express Olig2 but not Nkx2.2a. From these data, we conclude that, at stage of patterning rearrangement, Sulf2a function is again required to position dorsal boundary expression of the $\mathrm{Nkx} 2.2 \mathrm{a}$ domain, but also to ensure that a population of Olig2-expressing progenitors that do not activate Nkx2.2a are generated.

Together, these data highlight the key role of Sulf2a in assigning and maintaining neural progenitor identity at neurogenic and gliogenic stages.

Sulf2a regulates allocation of oligodendrocyte lineage cell fates. In zebrafish, newly specified OPCs, all expressing Olig2, represent a heterogeneous cell population in regard to Nkx2.2a expression and fate. While some OPCs express Nkx2.2a and differentiate rapidly as oligodendrocytes, others, that do not express $\mathrm{Nkx} 2.2 \mathrm{a}$, remain as non-myelinating OPCs ${ }^{40,41}$. Hereafter we will refer to these two OPC subsets as myelinating and non-myelinating OPCs, respectively. Whether these two OPC subsets emerge from Olig2 neural progenitors already distinguishable by differential Nkx2.2a expression remained an open question. Our data, highlighting the requirement of Sulf2a specifically for maintaining progenitors expressing only Olig2, led us to ask whether Sulf2a contributes specifically to generate the non-myelinating OPC subset. We thus examined the consequences of Sulf2a depletion on the development of the two distinct OPC populations by performing immunodetection of the pan-OPC marker Sox10 in $\operatorname{Tg}(n k x 2.2 a$ :mEGFP; olig2:DsRed2) embryos in which the two OPC subtypes can be distinguished by differential reporter expression ${ }^{36}$. As expected, at early gliogenic period (48 hpf), we found that the global population of OPCs, i.e. the Sox10+/olig2:DsRed2 + cells, was reduced in number either in sulf2aMO-injected or in sulf $2 a-/-$ embryos, decreasing by $40 \%$ compared to controls (Fig. $6 \mathrm{a}-\mathrm{e}$ ). We next 


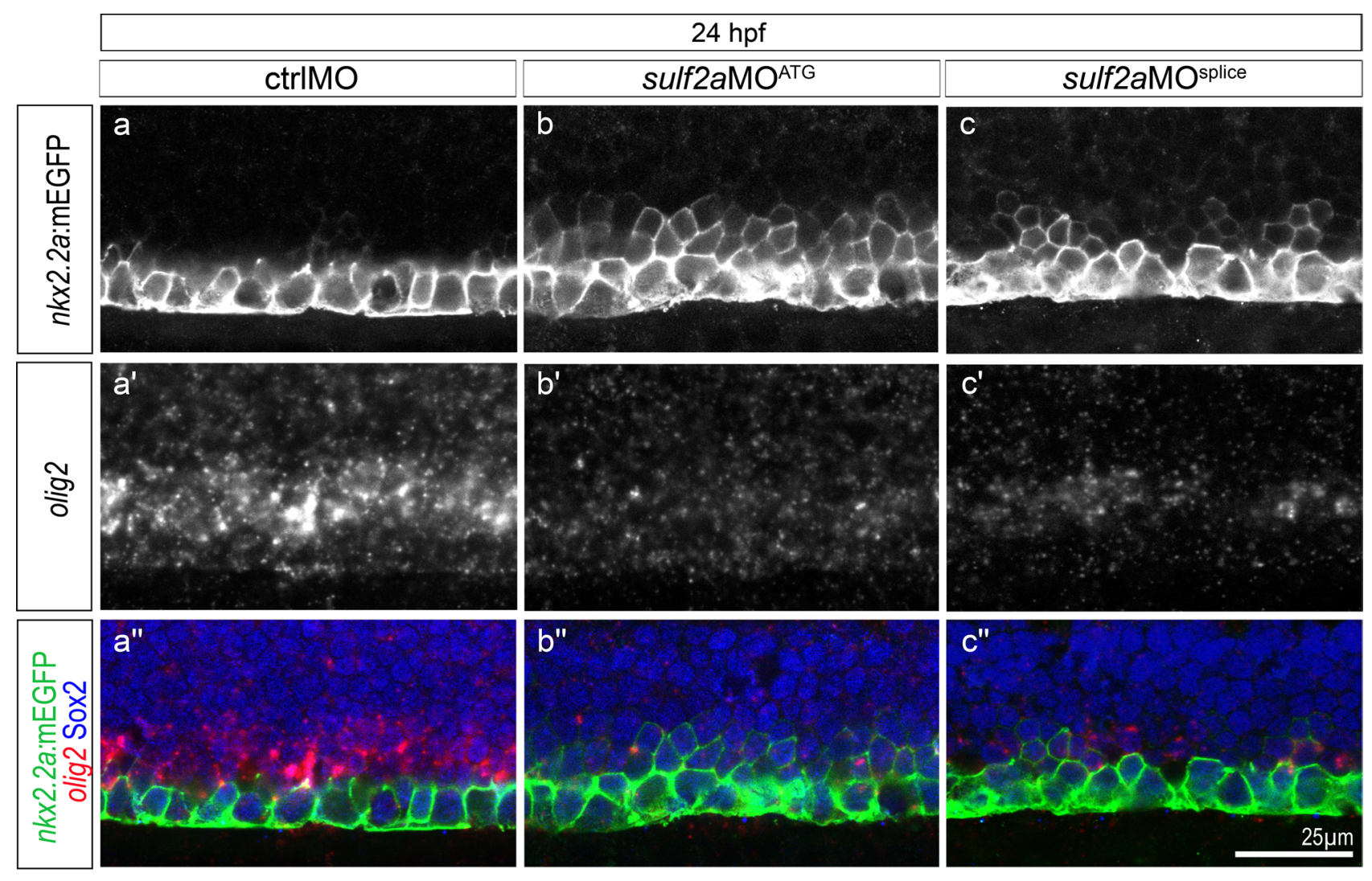

d

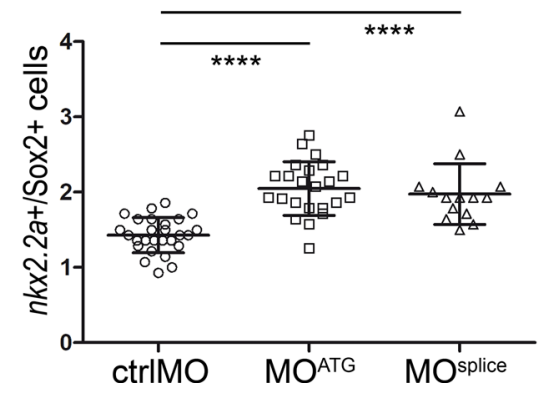

e

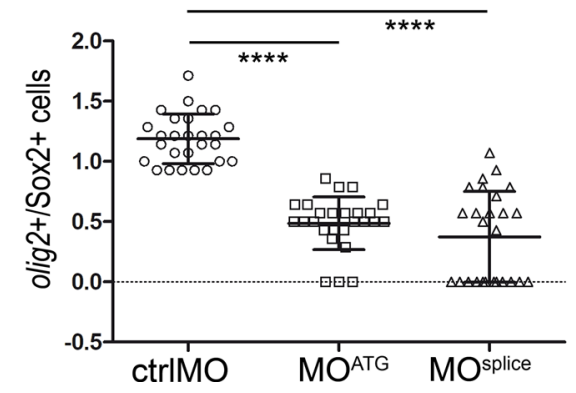

Figure 4. The Nkx2.2a/p3 domain is dorsally expanded at the expense of the Olig2/pMN domain in $24 \mathrm{hpf}$ sulf $2 a$-depleted embryos. Side views of $24 \mathrm{hpf}$ embryos. (a-c") Double detection of olig2 mRNA and Sox2 in $\mathrm{Tg}\left(n k x 2.2 a\right.$ :mEGFP) embryos injected with ctrlMO (a-a"), sulf2aMO $\mathrm{MTG}^{\mathrm{AT}}\left(\mathrm{b}-\mathrm{b}\right.$ ") or sulf $2 a \mathrm{MO}^{\text {splice }}$ (c-c"). Vertical sets present successively the $n k x 2.2 a$ :mEGFP signal (green in a" $\left.-c^{\prime \prime}\right)$, the olig2 mRNA staining (red in a" $-c^{\prime \prime}$ ) and the merged image together with Sox2 staining (blue in a"-c"). Note the dorsal expansion of the $n k x 2.2 a$ :mEGFP signal associated with a strong decrease in the olig2 signal in sulf2a morphant embryos. (d, e) Quantification of $n k x 2.2 a: \mathrm{mEGFP}+/$ Sox $2+(\mathrm{d})$ and olig2 + /Sox $2+(\mathrm{e})$ progenitors in embryos injected with ctrlMO $(\mathrm{n}=27)$, sulf $2 a \mathrm{MO}^{\mathrm{ATG}}(\mathrm{n}=14)$ and sulf $2 a \mathrm{MO}^{\text {splice }}(\mathrm{n}=23)$ from two independent experiments. Datasets were compared with Mann Whitney's test (two-tailed). Data are presented as a mean number of cells along the dorso-ventral axis per embryo \pm s.d $(* * * * \mathrm{p}<0.0001)$.

considered separately the myelinating (olig2:DsRed2 $+/ n k x 2.2 a$ :mEGFP + ) and the non-myelinating (olig2:DsR ed2+/nkx2.2a:mEGFP-) OPC subtypes. Quantification of these cells pointed to a decrease in number of both OPC populations but to different extent. In sulf $2 a$ morphants, non-myelinating OPCs were found decreased in number by $64 \%$ (Fig. 6a, b", g) while the myelinating population was reduced only by $31 \%$ compared to controls (Fig. 6a, b", f). A similar tendency was obtained analysing the two OPC subsets in sulf2a-/- embryos (Fig. 6c, d", $\mathrm{f}, \mathrm{g}$ ). Thus, the impaired development of OPCs observed in Sulf2a-depleted embryos is mostly attributable to a decreased number of OPCs that do not express Nkx2.2a.

These results, showing that Sulf2a plays a prominent role in promoting the development of non-myelinating OPCs, support the view that Sulf2a contributes to the early segregation of the two OPC lineages by preserving a pool of progenitors that only express Olig2. 
Sulf2a expression in neural progenitors prevents them to activate high-threshold response to Shh. Our results, pointing to a regulatory function of Sulf2a on limiting the dorsal extent of Nkx2.2a expression, highlight the key role of Sulf2a in maintaining Olig2-expressing progenitors at neurogenic and gliogenic stages. Regulation of olig2 and $n k x 2.2 a$ expression is known to depend on different levels of Shh signalling in zebrafish, $n k x 2.2 a$ requiring higher doses to be induced ${ }^{7,37,42,43}$. Thus, an attractive hypothesis was that Sulf2a acts by preventing activation of high Shh signalling in ventral neural progenitors. According to this possibility, $n k x 2.2 a+$ cells should not express sulf2a. To test this, we compared sulf $2 a$ and $n k x 2.2 a$ expression domains at 24 and $36 \mathrm{hpf}$ performing sulf $2 a$ fluorescent in situ hybridisation in $\operatorname{Tg}(n k x 2.2 a$ :mEGFP) embryos. We found that, at each stage, sulf2a and $n k x 2.2 a$ :mEGFP expression defined two distinct domains in the ventral developing spinal cord (Fig. $7 \mathrm{a}-\mathrm{d}$ ), thus confirming that expression of sulf $2 a$ is excluded from $n k x 2.2 a$-expressing cells. Noticeably, ventral boundary of the sulf2a + domain was found to abut dorsal boundary of the $n k x 2.2 a$ :mEGFP + domain at each stage (Fig. 7a-d). These observations suggested that, at $24 \mathrm{hpf}$, sulf $2 a$ expression overlaps the entire Olig2 + domain while it is not the case at $36 \mathrm{hpf}$, since, at that stage, the dorsal-most Nkx2.2a + cells are known to co-express Olig2. To clarify this, we analysed expression of sulf2a mRNA at $36 \mathrm{hpf}$ in $\mathrm{Tg}$ (olig2:GFP) embryos and found expression of sulf $2 a$ in a subset of Olig2 progenitors (Fig. 7e, f). Sulf2 2 expression being excluded from the Nkx2.2a-expressing domain, we conclude that these cells correspond to Olig2 progenitors that are not included in the $\mathrm{p}^{*}$ domain. Overall, these data, showing that sulf $2 a$ is excluded from progenitors that respond to high Shh signalling activity ( $\mathrm{Nkx} 2.2 \mathrm{a}+$ cells) but expressed in progenitors that do not activate high-threshold response to Shh (Olig2 + cells), support the view that Sulf2a might act at the surface of Olig2 + progenitors to reduce their sensitivity to Shh signalling. They moreover highlight differential expression of sulf2a in Olig2 progenitors at gliogenic stage.

An attractive hypothesis was thus that Sulf2 restricts the range of high-threshold response to Shh along the ventral to dorsal axis of the progenitor zone. To test this possibility, we used $\operatorname{Tg}$ (GBS-ptch2:EGFP) reporter line in which cells responding to Shh can be visualized by EGFP expression ${ }^{44}$. At $24 \mathrm{hpf}$, in control embryos, GBSptch2:EGFP expression was detected in the ventral neural tube in medial floor plate cells as well as adjacent progenitors that both express Sox2 (Fig. 8a,b). We interpreted the EGFP signal in medial floor plate cells as perdurance of the reporter protein since, while responsible for medial floor plate induction at earlier stages, Shh signalling is known to be inactive in these cells at $24 \mathrm{hpf}^{45,46}$. Moreover, EGFP was not detected at distance from the Nkx2.2a/p3 domain, indicating that the EGFP signal only allowed detecting the highest levels of Shh signalling. Having this in mind, we next examined EGFP expression in sulf2aMO-injected embryos. We found that the EGFP expression domain was enlarged and cell quantification confirmed a slight but significant increase in the number of EGFP + /Sox 2 + cells compared to controls (Fig. 8c-e), thus validating the role of Sulf2a in limiting the dorsal extent of high-threshold Shh signalling activity.

To bring additional support to the view that Sulf2a acts as a negative regulator of Shh signalling, we analysed expression of patched2, a as a marker for Shh response $e^{47}$. Fluorescent in situ hybridisation of patched 2 combined with Sox 2 immunodetection was performed at $24 \mathrm{hpf}$ and the relative fluorescent in situ signal was quantified along the ventral to dorsal axis of the neural tube (Fig. $8 \mathrm{f}-\mathrm{h}$ ). In control embryos, as previously reported ${ }^{48}$, we found graded expression of patched2, decreasing dorsally in neural progenitors (Fig. 8f,h). Noticeably, dorsally to the highest ventral signal, the intensity curve declines with a bending and then flattens dorsally, indicating that the response to Shh does not decrease linearly from the ventral region of the neural tube. Similarly, in sulf2aMO-injected embryos, patched 2 gradient progressively decreased dorsally (Fig. 8g,h). However, difference was observed in the shape of the curve which does bend but appeared decreasing dorsally in a linear manner (Fig. 8h). Comparison of patched 2 relative signal intensities further confirmed significant higher levels of patched 2 expression at the level of the intermediate neural tube in sulf2a-depleted embryos compared to controls (Fig. 8h), thus reflecting higher levels of Shh signalling activity in neural progenitors that should have expressed sulf2a. These results thus reinforce the view that sulf $2 a$ acts to limit response to Shh.

Importantly, we found that shh expression was not modified in sulf2a-depleted embryos (Supplementary Fig. 4), indicating that the influence of Sulf2a on Shh signalling activity does not depend on transcriptional regulation of the morphogen factor.

These data, showing that Sulf2a activity is required to downregulate Shh activity and in particular to limit the dorsal extent of high-threshold Shh response, highlight a functional relationship between Sulf2a and Shh, the enzyme acting by reducing the sensitivity of target cells to the Shh signal.

\section{Discussion}

In the ventral spinal cord, generation of distinct neural cell types is a spatio-temporally regulated process that mainly depends on the morphogenetic activity of Shh. During development, neural progenitors are exposed to varying concentrations of Shh which drive them to adopt specific transcriptional identities and eventually generate a particular neuronal or glial cell fate. We show here that Sulf2a contributes to assign specific neural identities to Shh-responding progenitors. We provide evidence that expression of sulf2a in Olig2 progenitors is essential to prevent highest levels of Shh signalling within these cells, thus allowing them to orient toward the production of, first MNs and, later on, of a particular subtype of OPC. Our data support a model in which Sulf2a spatially restricts expression of high-threshold Shh responsive genes to cells closest to the source of Shh (Fig. 9).

The first main finding of our work is that, in contrast to other vertebrates where the different Sulfs display overlapping expression domains in the developing spinal cord ${ }^{10,11,24-26}$, in zebrafish, a subset of spinal neural progenitors only expresses Sulf2a throughout neurogenesis and gliogenesis. Starting out from this observation, studying the role of Sulf2a in zebrafish represented a unique opportunity to investigate the still debated question of whether Sulf proteins fulfill redundant functions or contribute differentially to spinal cord development ${ }^{9,11,26}$. During the neurogenic phase, which takes place between 10 and $30 \mathrm{hpf}$ in the ventral zebrafish spinal $\operatorname{cord}^{28}$, we 


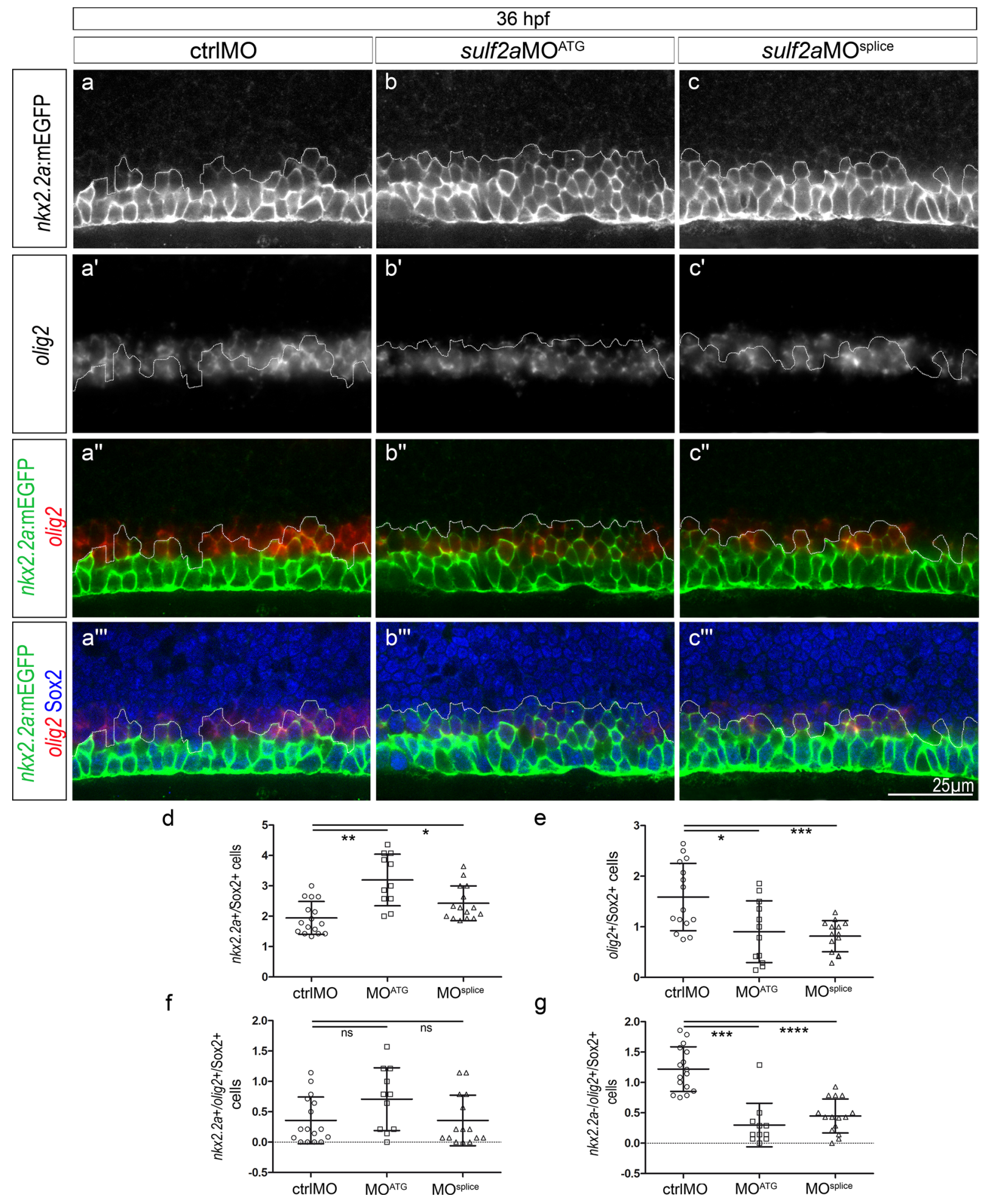


4 Figure 5. Sulf2a depletion does not affect formation of the Nkx2.2a/Olig2-co-expressing $\mathrm{p}^{*}$ domain but reduces the number of progenitors that only express Olig2 at the onset of gliogenesis. Side views of 36 hpf embryos. (a-c'"): Double detection of olig2 mRNA and Sox2 in Tg(nkx2.2a:mEGFP) embryos injected with ctrlMO (a-a"'), sulf2aMO ${ }^{\text {ATG }}$ (b-b"')

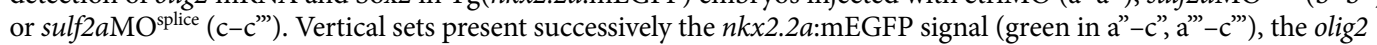
mRNA staining (red in a" $-c$ ", a"' $c$ "'), the merged image of $n k x 2.2 a$ :mEGFP and olig2 signals and the merged image together with Sox2 staining (blue in a"'-c'”). Dotted line represents the dorsal limit of $n k x 2.2 a$ :mEGFP-expressing domain. (d-g) Cell quantification performed in embryos injected with ctrlMO $(\mathrm{n}=16)$, sulf2aMO ${ }^{\mathrm{ATG}}(\mathrm{n}=11)$ and sulf $2 a \mathrm{MO}^{\text {splice }}(\mathrm{n}=15)$ from three independent experiments. Cell counts in $(\mathrm{d}, \mathrm{e})$ correspond to the population of Sox $2+$ progenitors expressing either $n k x 2.2 a$ :mEGFP (d) or olig2 (e). Quantifications in (f,g) correspond to Sox $2+$ progenitors that either co-express olig2 and $n k x 2.2 a$ :mEGFP (f) or express olig2 mRNA but not $n k x 2.2 a$ :mEGFP (g). Except in e where datasets were compared with Student's $t$-test (unpaired two-tailed), datasets were compared with Mann Whitney's test (two-tailed). Results are presented as a mean number of cells along the dorso-ventral axis per embryo \pm s.d $\left({ }^{*} \mathrm{p}<0,05,{ }^{* *} \mathrm{p}<0,005,{ }^{* * *} \mathrm{p}<0.001,{ }^{* * *} \mathrm{p}<0.0001\right)$.

showed that Sulf2a prevents excess production of V3 interneurons and fosters MN generation. Later on, from $36 \mathrm{hpf}$, Sulf2a, while not essential for OPC production, has proven to play a key role in triggering generation of non-myelinating OPCs. This markedly contrasts with the function of Sulf1 which stimulates V3 interneuron production, has no impact on MN generation and proves to affect more OPC generation when depleted ${ }^{7}$. Thus, our work makes clear that Sulf2a and Sulf1 exert differential functions in the ventral spinal cord.

We next provide evidence that Sulf2a is part of the regulatory process that provides positional identity to ventral neural progenitors. Specifically, Sulf2a is required, either at neurogenic or gliogenic stage, to maintain a pool of progenitors that express Olig2 but not Nkx2.2a. At neurogenic stages, sulf2a expression defines the ventral boundary of the Olig $2 / \mathrm{pMN}$ domain and acts to prevent dorsal expansion of Nkx2.2a expression within this domain. In agreement with the well-known repressive activity of Nkx2.2a on olig2 expression at this stage $\mathrm{e}^{17,20,31,49}$, dorsal misexpression of Nkx2.2a observed in Sulf2a-depleted embryos is accompanied by a marked reduction in the number of Olig2 progenitors. Thus, the progenitor fate change caused by Sulf2a depletion is sufficient to explain the overproduction of $\mathrm{V} 3$ interneurons at the expense of $\mathrm{MN}$ development observed in these embryos. However, also likely contributing to $\mathrm{V} 3$ interneuron overproduction, is the presence of $\mathrm{Nkx} 2.2 \mathrm{a} / \mathrm{p} 3$ progenitors that persist in Sulf2a-depleted embryos at gliogenic stage. Indeed, in contrast to the wild-type situation, in these embryos, we observed that the Nkx2.2a/p3 domain extends dorsally to the Shh-expressing domain (lateral floor plate).

A somewhat similar but more complex situation was found regarding progenitor identity regulation by Sulf $2 \mathrm{a}$ at the onset of gliogenesis where sulf $2 a$ expression, still excluded from the Nkx2.2a-expressing domain, thus from the $\mathrm{p}^{*}$ domain, is detected in a subset of Olig2 progenitors. Consistently, the enzyme appears dispensable for formation of the $\mathrm{p}^{\star}$ domain but required for proper development of progenitors that only express Olig2. How Sulf2a might control development of this particular progenitor population however remains elusive. Since Nkx2.2a no more represses Olig2 at initiation of gliogenesis, dorsal misexpression of Nkx2.2a cannot provide a simple explanation to the loss of Olig2 progenitors caused by Sulf2a depletion as it is the case at neurogenic stage. An alternative explanation might be that Sulf2a plays a role in progenitor recruitment, a process that occurs at initiation of gliogenesis and during which progenitors positioned dorsally to the pMN domain descend to this domain and only then initiate Olig2 expression, a process known to depend on Shh signalling ${ }^{41,50}$.

Quite apart from the question of how Sulf2a regulates neural patterning, our data shed also new light on the still open question of the origin and relationship of progenitors that give rise to the two OPC subtypes in zebrafish. Two distinct types of spinal OPCs, ones expressing Nkx2.2a and differentiating rapidly as oligodendrocytes and others, not expressing Nkx2.2a and remaining as non-myelinating OPCs have recently being shown to arise from distinct Olig2 progenitor pools ${ }^{40,41}$. However, how these OPC populations, heterogeneous with respect to $\mathrm{Nkx} 2.2 \mathrm{a}$ expression and fate, are generated remained elusive. Our data, showing that Sulf2a depletion preferentially affects generation of both non-myelinating OPCs and Olig2 progenitors that do not express $\mathrm{Nkx} 2.2 \mathrm{a}$, strongly support the view of a lineage relationship between these cells. In apparent contradiction with this proposal, Nkx2.2a-expressing OPCs are also affected, although more moderately, by Sulf2a depletion despite the presence of a correct number of Olig $2 / \mathrm{Nkx} 2.2 \mathrm{a}$-co-expressing progenitors. Since neuronal signals and activity can alter OPC development ( $\mathrm{see}^{51}$ for review), this result can be potentially explained by the aberrant neuronal populations generated upon Sulf2a depletion.

Finally, examination of Shh signalling activity upon Sulf2a depletion together with the well-known dependence of Olig2 and Nkx2.2a expression on differential Shh thresholds ${ }^{37,42,43}$ are consistent with a role of Sulf2a in attenuating Shh activity at the surface of Olig2 progenitors. Consistently, development of the non-myelinating OPC subset, which is particularly affected by Sulf2a depletion, depends on lower doses of Shh as compared to myelinating $\mathrm{OPCs}^{41}$. Our work thus brings support to an essential role of Sulf2a-dependant HS sulfation in regulating Shh signal reception. This is in line with previous reports showing that the sulfation level of glypicans, a family of GPI-anchored HSPGs acting either as co-receptors or as competitors of Shh binding to its receptor Patched, is an important level of regulation of their function at the surface of Shh receiving cells ${ }^{52-55}$. Importantly, this work reveals that Sulf2a plays an opposite role than Sulf1 which stimulates Shh signalling in the developing spinal $\operatorname{cord}^{7-10}$. The differential expression of Sulf2a in neural progenitors and of Sulf1 in Shh-producing cells indicates that Sulfs can fulfill opposite functions on the very same signalling cascade by acting either at the source or in receiving cells. This is reminiscent to the dual function of DSulf1, the unique Sulfatase gene in Drosophila, 

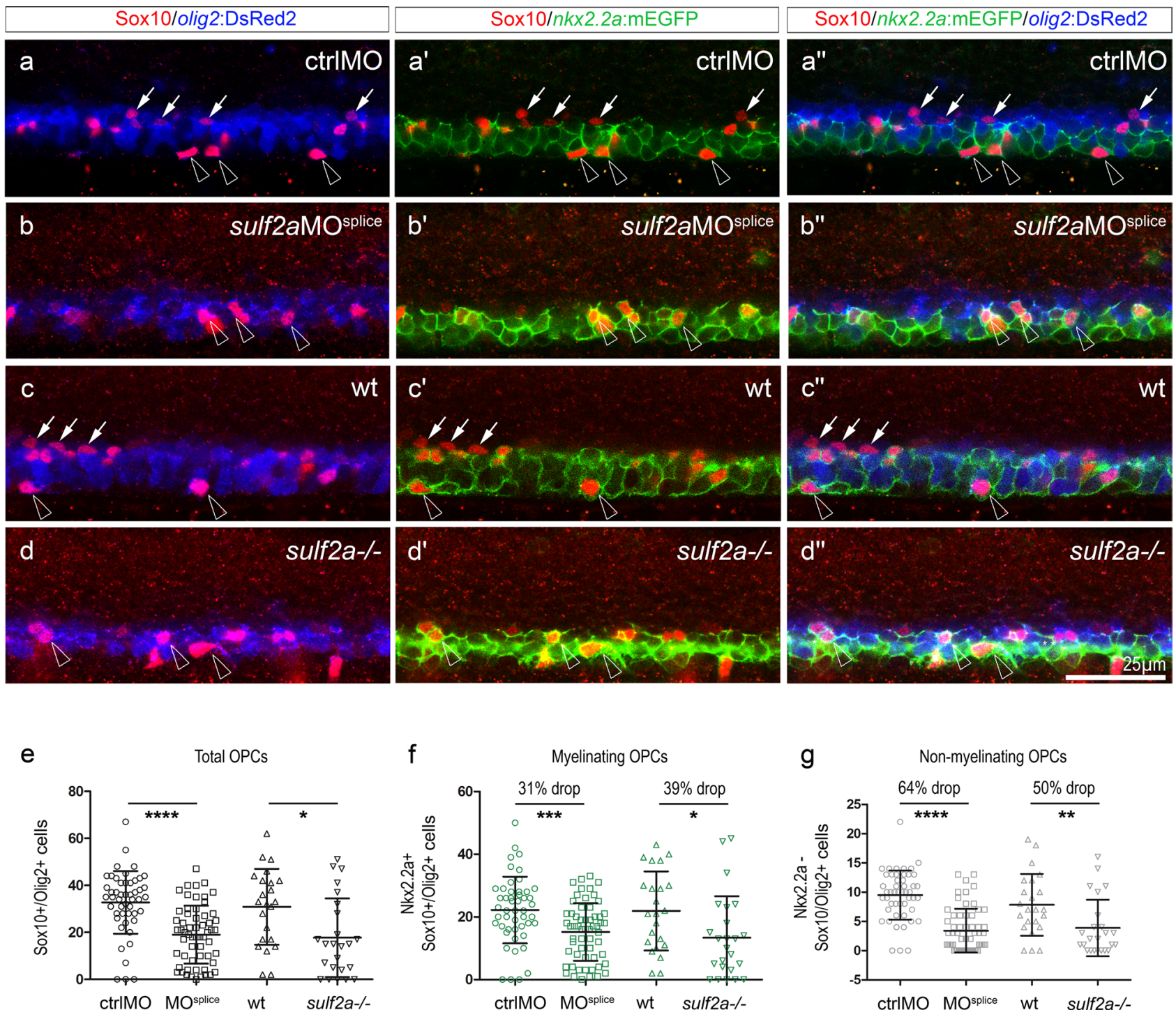

Figure 6. Sulf2a depletion causes a preferential deficit of non-myelinating OPCs. Side views of 48 hpf embryos. (a-d") Immunodetection of Sox10 in Tg(nkx2.2a:mEGFP; olig2:DsRed2) embryos injected with ctrlMO (a-a") or sulf $2 a \mathrm{MO}^{\text {splice }}$ (b-b") and wild-type (c-c") or mutant for sulf $2 a$ (d-d"). Horizontal sets present successively the Sox10 staining (red) in combination with the olig2:DsRed2 signal (blue, a-d), with the $n k x 2.2 a$ :mEGFP signal (green, a'-d') and the merged image of the three signals (a"-d"). White arrows point to non-myelinating OPCs (Nkx2.2a:mEGFP-/olig2:DsRed2 +/Sox10 + cells) while open arrowheads indicate myelinating OPCs (Nkx2.2a:mEGFP + /olig2:DsRed2 +/Sox10 + cells). (e-g) Quantification of OPCs in embryos injected with ctrlMO $(\mathrm{n}=70)$ or sulf2a $\mathrm{MO}^{\text {splice }}(\mathrm{n}=59)$ and in wild-type $(\mathrm{n}=23)$ or sulf $2 a-/-(\mathrm{n}=24)$ embryos from five and three independent experiments, respectively. Cell counts in (e) correspond to the total number of OPCs co-expressing Sox10 and olig2:DsRed2. Quantifications of $n k x 2.2 a$ :mEGFP +/myelinating OPCs (f) and $n k x 2.2 a$ :mEGFP-/non-myelinating OPCs (g) were presented separately. Datasets were compared with Mann Whitney's test (two-tailed). Data are presented as mean number of cells per embryo \pm s.d $\left({ }^{*} \mathrm{p}<0.05,{ }^{* *} \mathrm{p}<0.01\right.$, $\left.{ }^{* * *} \mathrm{p}<0.0005,{ }^{* * * *} \mathrm{p}<0.0001\right)$.

which is expressed both in Hh-producing and -receiving compartments where it exerts a positive and a negative regulatory function on Hh signalling, respectively ${ }^{56}$.

Overall, our work identifies Sulf2a as a crucial regulator of neural cell diversification within the ventral developing spinal cord through a negative action on Shh signalling. Sulf2a contributes to pattern gene expression in neural progenitor cells, sustaining low-threshold response to Shh morphogen at the two critical periods of neuronal and glial production. This work not only unravels a novel function for Sulf2a but also highlights how extracellular sulfatases contribute in different ways to a given signalling pathway depending on their spatial expression in a complex tissue. 


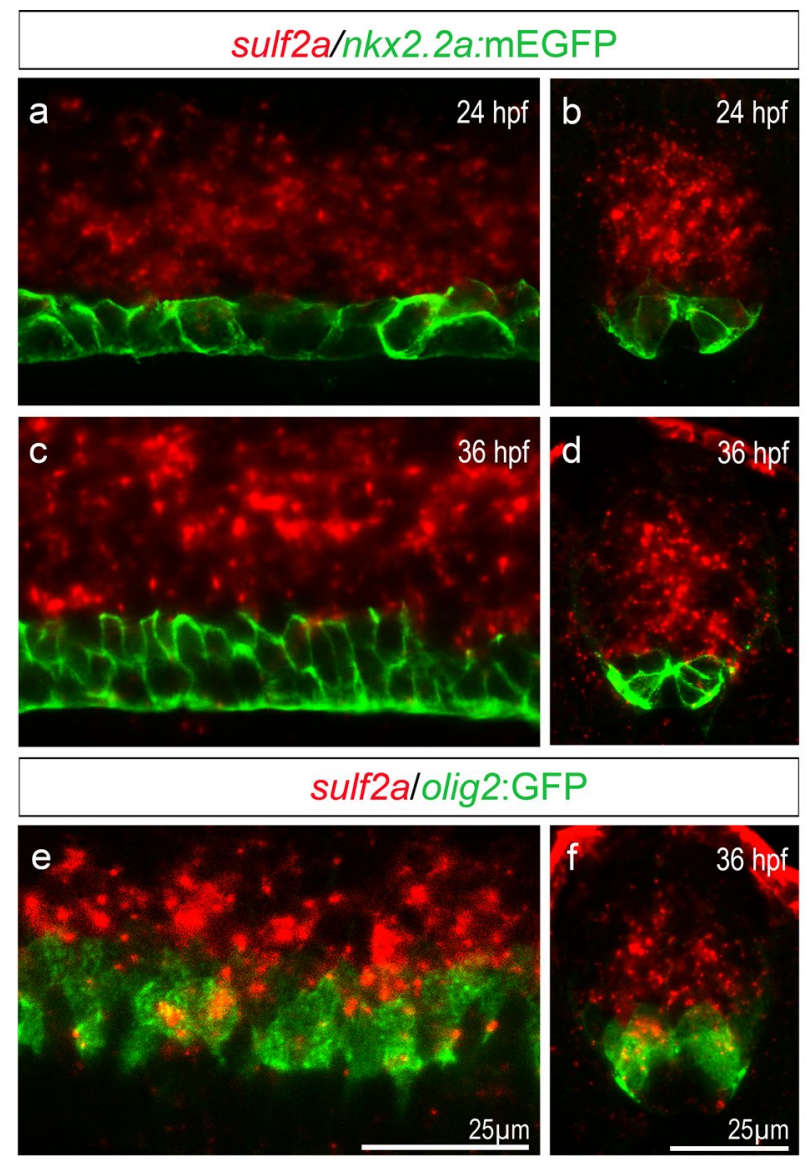

Figure 7. Sulf $2 a$ is excluded from Nkx2.2a-expressing progenitors but expressed, at gliogenic stage, in a subset of Olig2 progenitors. Side views (a,c,e) and transverse sections (b,d,f) of $24 \mathrm{hpf}(\mathrm{a}, \mathrm{b})$ and $36 \mathrm{hpf}(\mathrm{c}-\mathrm{f})$ embryos. (a-f) Detection of sulf2a mRNA (red) in Tg(nkx2.2a:mEGFP) embryos (green, a-d) or in Tg(olig2:GFP) embryos (green, e,f). Images show representative examples of at least three embryos. Note that sulf $2 a$ expression dorsally abuts the $n k x 2.2 a$ :mEGFP-expressing domain both at 24 and $36 \mathrm{hpf}(\mathrm{a}-\mathrm{d})$ and is heterogeneously expressed within the population of Olig2 progenitors at $36 \mathrm{hpf}(\mathrm{e}, \mathrm{f})$.

\section{Methods}

Animals and fish lines. Adult fishes were handled in a facility certified by the French Ministry of Agriculture (approval number A3155501). The project has received an agreement number APAFIS\# 2017061313143443 $\# 10204$. All efforts were made to minimize the number of animals used and their suffering, in accordance with the guidelines from the European directive on the protection of animals used for scientific purposes (2010/63/ UE) and the guiding principles from the French Decret 2013-118.

The following transgenic lines were used to visualise OPCs: $\operatorname{Tg}$ (olig2:EGFP) $v u 12^{35}, \operatorname{Tg}$ (olig2:dsRed2) $v u 19^{36}$ and $\operatorname{Tg}(n k x 2.2 a: \mathrm{mEGFP}) v u 17^{57}$. Shh signalling activity was assessed using the $\operatorname{Tg}($ GBS-ptch2:EGFP) transgenic line $e^{44}$.

Generation of sulf2a mutant by Crispr/Cas9 technology and genotyping. SgRNA targeting the exon 11 of sulf $2 a$ gene was designed using the CRISPOR selection tool ${ }^{58}$. The following primers were purchased from Integrated DNA Technologies (IDT): 5' TAGGCTTCCTCTTGAGAGGCGCTG 3' and 5' AAACCAGCG CCTCTCAAGAGGAAG 3', annealed and cloned into a pDR274 host vector (Addgene). After sequence-based selection, this vector was linearized and used as a template for producing RNAs by in vitro transcription (T7 mMessage mMachine, Ambion). The obtained sgRNA solution was mixed with Cas9 nuclease (NEB Cas9 Nuclease, S.pyogenes $20 \mathrm{mM}, \mathrm{M} 0386 \mathrm{M})$ at $45 \mathrm{ng} / \mu \mathrm{l}$ final concentration and used for microinjection in $\mathrm{Tg}(\mathrm{Olig} 2: \mathrm{GFP})$ one-cell stage embryos. F0 individuals carrying mutations were screened by sequencing PCR products using the following oligonucleotides: 5' TTCCTCTGTGCAGAAGTGGC 3' and 5' TCAATCTGACAACCTGCGCT 3'. F0 fish positive for mutation was outcrossed to wild-type fish. F1 offsprings were thus screened by sequencing PCR products using the above primers to confirm germline transmission, which led to identifying an indel mutation (16 nucleotide deletion and 9 nucleotide insertion) generating a STOP codon at position 519. F1 carrier fish was selected and crossed to wild-type fish in order to generate F2 individuals that were further used to produce the subsequent generations of embryos for phenotypic analysis. Embryos were obtained by crossing heterozygous fish and were further individually genotyped before analysis. Genotyping was performed from genomic DNA extracted from tail clipping for adults or from dissected heads from embryos, using the DirectPCR Lysis Reagent 


\section{GBS-patched2:EGFP/Sox2}

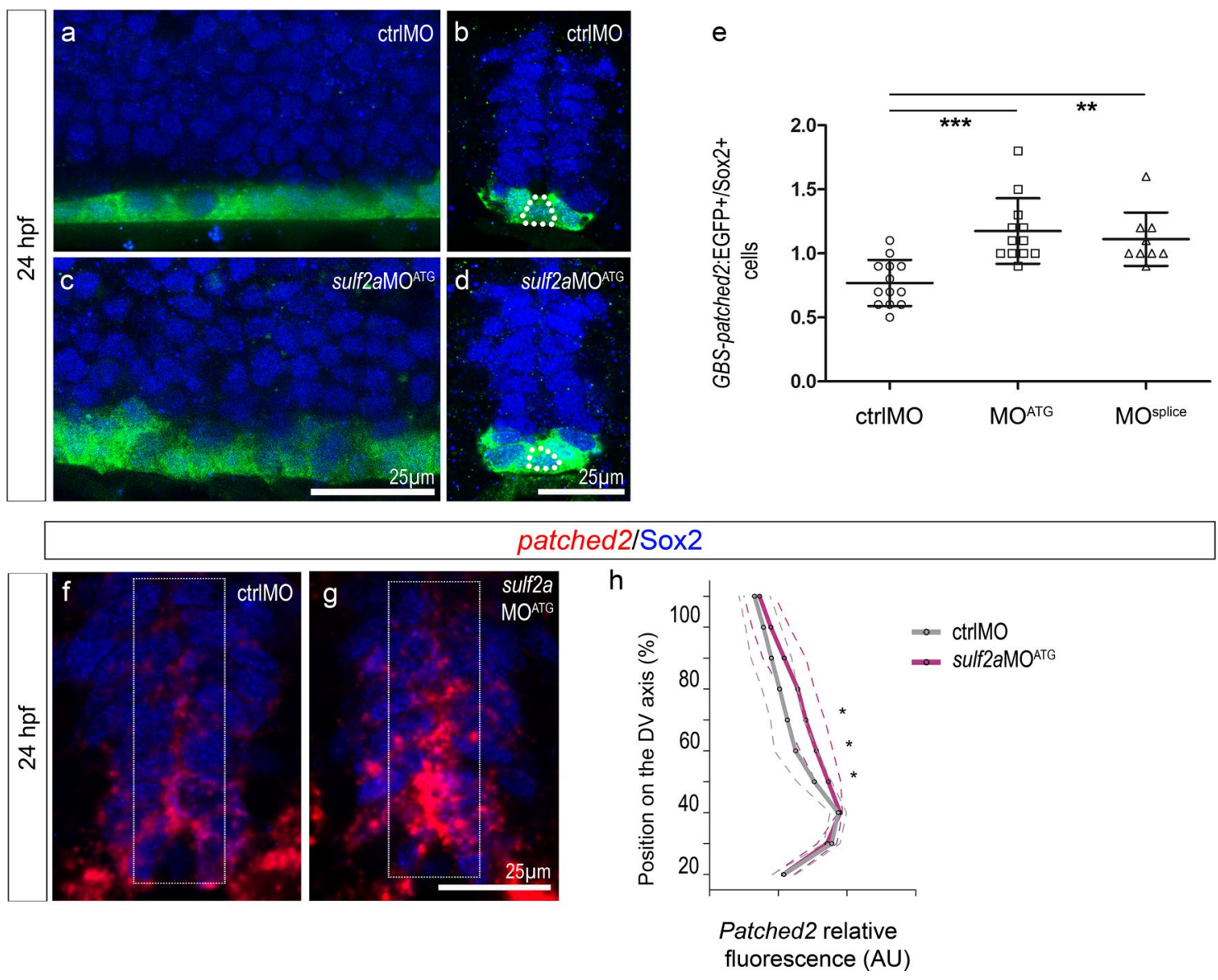

Figure 8. Sulf $2 a$ restricts dorsal extent of the high-threshold Shh response without affecting expression of shh. Side views $(\mathrm{a}, \mathrm{c})$ and transverse sections (b,d,f,g) of 24 hpf embryos. a-d: Immunostaining of Sox 2 in $\mathrm{Tg}\left(\right.$ GBS-ptch2:EGFP) embryos injected with ctrlMO (a,b) or sulf2aMO ${ }^{\mathrm{ATG}}$ (c,d). Dotted lines in b and d delineate the medial floor plate. Note the dorsal expansion of the GBS-ptch2:EGFP signal in sulf2a morphants. (e) Quantification of GBS-ptch2:EGFP + /Sox 2 + cells in embryos injected with ctrlMO ( $\mathrm{n}=13)$, sulf2aMO ${ }^{\mathrm{ATG}}$ $(\mathrm{n}=12)$ or sulf $2 a \mathrm{MO}^{\text {splice }}(\mathrm{n}=9)$ from two independent experiments. Datasets were compared with Mann Whitney's test (two-tailed). Data are presented as mean number of cells along the dorso-ventral axis on each side of the lumen per embryo \pm s.d $\left({ }^{* *} \mathrm{p}<0.0005,{ }^{* *} \mathrm{p}<0.005\right)$. (f-h) Double detection of patched $2 \mathrm{mRNA}$ (red, $f, g$ ) and Sox2 (blue, $f, g)$ in embryos injected with ctrlMO (f, $n=5)$ or sulf $2 a \mathrm{MO}^{\mathrm{ATG}}(\mathrm{g}, \mathrm{n}=5)$. Patched2 relative fluorescence intensity in neural progenitors (white frame in $\mathrm{f}, \mathrm{g}$ ) was quantified along the dorso-ventral (DV) axis (grey line for ctrlMO and purple for sulf $2 a \mathrm{MO}^{\mathrm{ATG}}$ ). Datasets were compared with two-way ANOVA test. Data are presented in arbitrary units of fluorescence (AU; mean values \pm s.d) along the DV axis per section $\left({ }^{\star} \mathrm{p}<0.005\right)$.

(Viagen), supplemented with Proteinase K. PCR fragments were amplified (Go Taq, Promega) using the oligonucleotides mentioned above and subsequently sequenced by Eurofins genomics. Genotypes were interpreted from obtained chromatographs.

Microinjection experiments. Microinjections were carried out in one or two-cell stage embryos using a pressure microinjector (Eppendorf FemtoJet). The following Morpholinos (MOs) (Gene Tools, LLC) were used: sulf $2 a \mathrm{MO}^{\text {ATG }}$ (5'-GACCTTAACCAGTGCTCCACCTCCC-3', $\left.80 \mu \mathrm{M}\right)$, sulf2aMO ${ }^{\text {splice }}$ (5'- GAAACTATACTG TGACCCACTCTTC-3', $1000 \mu \mathrm{M}$ ) and standard control MO targeting the human $\beta$-globin (ctrlMO, $1000 \mu \mathrm{M})$. For rescue experiments, sulf2a mRNA was synthetised with T7 mMessage machine kit (Ambion), from linearized plasmid containing zebrafish full length cDNA (Sulf2a-ODa35785C-pcDNA3.1(+); Genscript). Sulf2a

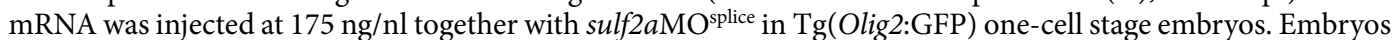
were injected with a $0.1 \mathrm{nl}$ solution. Data presented for each MO are combined for at least two independent experiments.

RT-PCR experiments. Total RNAs were extracted using Trizol from 20 injected embryos at 24 hpf for each condition. $500 \mathrm{ng}$ of RNAs were used to perform reverse transcription following provider guidelines (SuperScript II, Invitrogen). Resulting cDNA was used as template for PCR reaction using a forward oligonucleotide 
hybridizing in exon $115^{\prime}$ GCTGCCAACAGAGGACAGAT 3 ' and a reverse oligonucleotide hybridizing in exon $125^{\prime}$ TTGAGGTTGAGGGGACGGTA 3' of sulf2a gene. PCR product size was analysed on agarose gel, sulf2a spliced mRNAs give rise to a $221 \mathrm{bp}$ band while unspliced forms generate a $2430 \mathrm{bp}$ size PCR product (Supplementary Fig. 1a,b).

Staining procedures. Fixation and storage. Embryos were raised and staged according to standard protocols ${ }^{59}$. Staged embryos were released from the chorion using forceps and placed in a $3.7 \%$ formaldehyde solution overnight at $4{ }^{\circ} \mathrm{C}$ for in situ hybridisation or $1 \mathrm{~h}$ at room temperature for immunostainings. Then embryos were dehydrated through a methanol series and stored in $100 \%$ methanol at $-20^{\circ} \mathrm{C}$.

Immunodetection. After rehydration, embryos were treated with $0.25 \%$ Trypsin/EDTA (Sigma) at $37^{\circ} \mathrm{C}$ from 1 to $7 \mathrm{~min}$ according to their developmental stage. Subsequent incubations and washes were performed in PBS containing $0.8 \%$ Triton. The following primary antibodies were used for an overnight incubation at $4{ }^{\circ} \mathrm{C}$ : rabbit anti-Sox10 (1/1000, Genetex), rabbit anti-Sox2 (1/1000, Genetex), mouse anti-Islet1-2 (1/10, DHSB, 39.4D5). GFP and DsRed2 expression in transgenic embryos was detected using chick anti-GFP $(1 / 1000$, Abcam) and rat anti-RFP (1/1000, Chromotek) primary antibodies, respectively. Subsequently, Alexa fluor 488, 555 and 647-conjugated secondary goat antibodies (anti-chicken antibody was purchased from Abcam and all others from Molecular probes) were used at $1 / 500$ for $6 \mathrm{~h}$ at room temperature. For triple immunostainings, we sequentially performed double and single immunodetections. Embryos were mounted in mowiol for subsequent observation.

Wholemount in situ hybridisation. The following RNA probes were used: sulf $2 a$ and sulf $2 b^{27}$, olig $2^{37}, \operatorname{sh} h^{60}$, $\operatorname{sim} 1 a^{61}$. In all cases, washes were performed in PBS containing $0.5 \%$ Tween (PBT) and the hybridisation step was achieved over $24 \mathrm{~h}$.

Single in situ hybridisation. Single in situ hybridisation was adapted from Xu et al. ${ }^{62}$ with substantial changes in the hybridisation solution. Briefly, rehydrated embryos were permeabilized with a proteinase $\mathrm{K}$ treatment $(10 \mu \mathrm{g} / \mathrm{ml})$ from 1 to $45 \mathrm{~min}$ according to developmental stage, washed in PBT and then re-fixed $20 \mathrm{~min}$ in a $3.7 \%$ formaldehyde $/ 0.1 \%$ glutaraldehyde solution. After PBT washes, embryos were placed $3 \mathrm{~h}$ at $65{ }^{\circ} \mathrm{C}$ in the hybridisation mix containing 50\% formamide, 5\% Dextran, 1.,3X SSC pH5. $5 \mathrm{mM}$ EDTA pH8, $50 \mu \mathrm{g} / \mathrm{ml}$ tRNA yeast, $0.2 \%$ Tween, $0.5 \%$ CHAPS, $0.1 \mathrm{mg} / \mathrm{ml}$ heparin. Then, hybridisation mix containing the diluted digoxygenin-labelled probe was applied over $24 \mathrm{~h}$ at $65^{\circ} \mathrm{C}$. Subsequently, embryos were washed several times in hybridisation mix at $65{ }^{\circ} \mathrm{C}$. After a wash in a $50 \%$ dilution of hybridisation solution in TBST ( $25 \mathrm{mM}$ Tris Base, $140 \mathrm{mM} \mathrm{NaCl}, 2.7 \mathrm{mM} \mathrm{KCl}, 0,1 \%$ Tween) at $65^{\circ} \mathrm{C}$, immunodetection was achieved with anti-digoxygenin antibody (Roche) diluted at $1 / 2000$ in TBST/2\% Blocking reagent (Roche)/20\% Goat serum. NBT/BCIP color reaction was started after TBST washes and monitored under a dissecting microscope. Embryos were mounted in mowiol for subsequent observation.

Double fluorescent in situ hybridisation. Protocol was adapted from Denkers et al. ${ }^{63}$. After proteinase K permeabilisation, embryos were post-fixed $30 \mathrm{~min}$ with a $3.7 \%$ Formaldehyde solution at room temperature. Fluorescein and digoxygenin labelled probes were incubated together and revealed sequentially: HRP-conjugated antibody against fluorescein (Roche, 1/2000) was incubated overnight and revealed with Cyanine3 TSA (Perkin Elmer). Subsequently, peroxidase was inactivated by $45 \mathrm{~min}$ incubation in $1 \% \mathrm{H} 2 \mathrm{O} 2$. After three washes, HRPconjugated antibody against digoxygenin (1/1000; Roche) was applied overnight and revealed with 488-TSA solution (Perkin Elmer). Embryos were mounted in mowiol after at least $1 \mathrm{~h}$ of PBT washes.

Fluorescent in situ hybridisation coupled with immunodetection. Fluorescent in situ hybridisation coupled with immunodetection was adapted from Denkers et al. ${ }^{63}$. After hybridisation with the olig2 digoxygenin-labelled probe, HRP-coupled anti-digoxygenin antibody (1/1000, Roche) was incubated together with chick anti-GFP and rabbit anti-Sox 2 primary antibodies overnight. Subsequently, Alexa 488-conjugated goat anti-chick and Alexa 647-coupled goat anti-rabbit secondary antibodies were applied $5 \mathrm{~h}$ at room temperature. After PBT washes, detection of the HRP-coupled antibody was achieved by applying Cyanine3 TSA reagent (Perkin Elmer) $30 \mathrm{~min}$ at room temperature. Embryos were mounted in mowiol after at least $1 \mathrm{~h}$ of PBT washes.

Sectioning. After staining, embryos were prepared as described in Andrieu et al. ${ }^{64}$. Briefly, embryos are incubated overnight in Phosphate Buffer (PB)/15\% sucrose at $4{ }^{\circ} \mathrm{C}$. Then, embryos were incubated $2 \mathrm{~h}$ in $\mathrm{PB} / 15 \%$ sucrose $/ 7.5 \%$ gelatine at $42{ }^{\circ} \mathrm{C}$ and transferred into a in $\mathrm{PB} / 15 \%$ sucrose $/ 7.5 \%$ coated dish. Individual blocks were prepared under a dissecting microscope, frozen in isopentane (Sigma, 615838) at $-80^{\circ} \mathrm{C}$ and stored at $-80^{\circ} \mathrm{C}$. Cryostat Leica CM1950 was used to generate $20 \mu \mathrm{m}$ thick sections. Slices were then incubated $20 \mathrm{~min}$ in PB at $42^{\circ} \mathrm{C}$ to remove gelatin and mounted in mowiol for subsequent observation.

Imaging, cell counting and statistical analysis. In situ hybridisation images were collected with Nikon digital camera DXM1200C/Nikon eclipse 80i microscope. Fluorescence photomicrographs were collected on Leica SP8 confocal microscope and were always represented as single optical plane sections. Images were processed (size adjustment, luminosity and contrast, and merging or separating layers) using Adobe Photoshop CS2 (Adobe). In all experiments, cell counts were performed in a defined window positioned along the antero-posterior axis of the spinal cord between somites 14 and 18. Provided data are the average of at least nine 
a

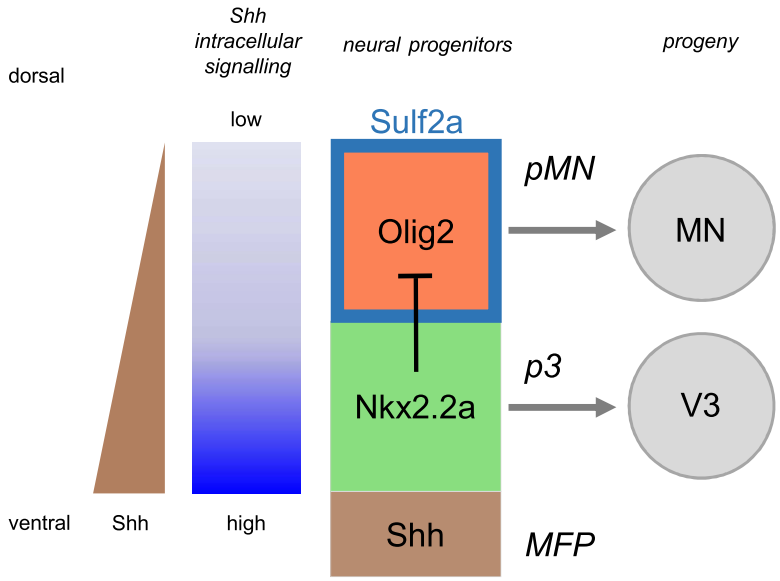

$a^{\prime}$

Sulf2a loss-of-function

Shh

intracellular neural progenitors progeny
signalling

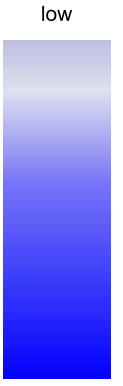

high

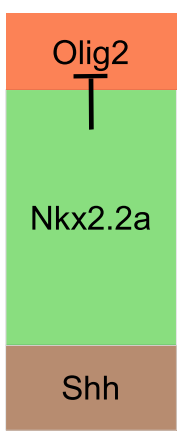

$p M N$

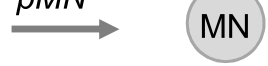

p3

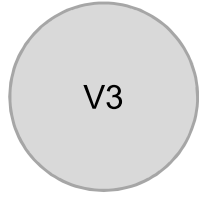

MFP

\section{$36 \mathrm{hpf}$}

b

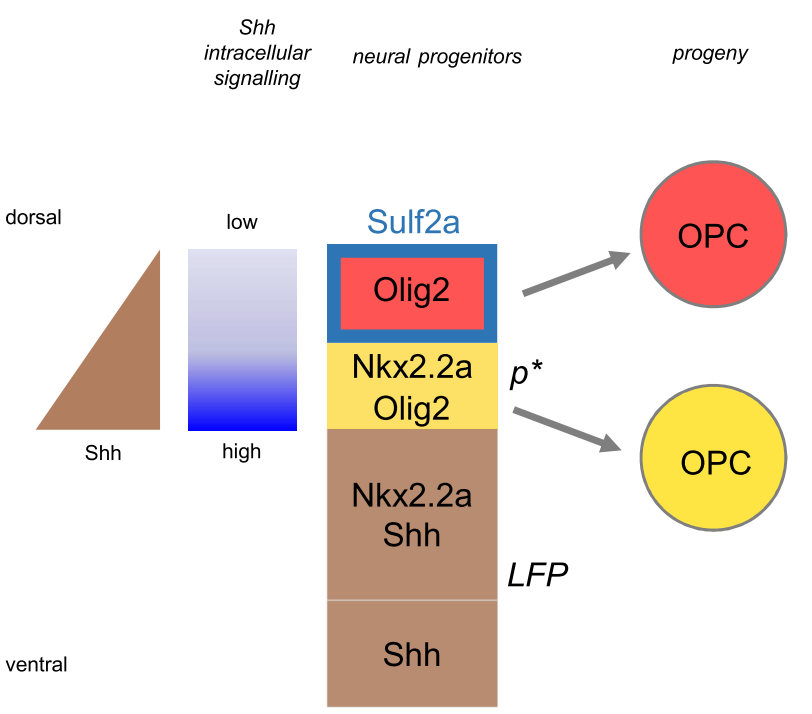

b'

Sulf2a loss-of-function low

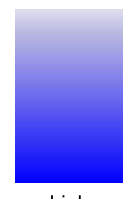

high

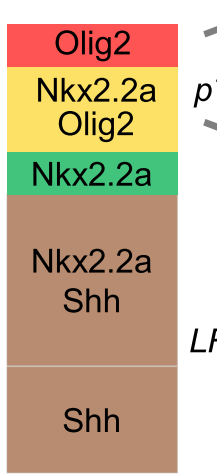

embryos per condition from at least two independent experiments. Numbers of individuals (n) are indicated in figure legends. Cell counts of sim $1 a+$ cells detected by in situ hybridisation in Fig. 2 were performed by direct observation under the microscope using a $40 \times$ objective, within a $300 \mu \mathrm{m}$ window. For fluorescent stainings, cell counts were performed from z-stacks collected on the entire spinal cord depth and using the Multipoint tool on Image J software. Cell counts of OPCs in Figs. 3 and 6 were performed in a $212 \mu \mathrm{m}$ window. Motor neurons in Supplementary Fig. 1 were counted in a $123 \mu \mathrm{m}$ window. To get information on the dorso-ventral extension of progenitor domains (Figs. 4, 5 and 8), we adapted a specific cell counting method. On a z-stack encompassing a $145 \mu \mathrm{m}$ window, straight lines, distant from $20 \mu \mathrm{m}$, were drawn perpendicular to the antero-posterior axis of the spinal cord. Along each of these lines, neural progenitors, marked by Sox 2 expression, were counted. Counts were performed on only one side of the lumen which is easily recognisable on z-stacks. For each embryo, the five cell counts were used to calculate an average value which was further used to perform statistical analyses. Quantifications of patched2 fluorescent signal (Fig. 8) were carried out on $z$ projections collected at identical 
4Figure 9. Model for Sulf2a function. Scheme showing Sulf2a and Shh-dependent gene expression in ventral neural progenitors during neuronal production ( $\left.24 \mathrm{hpf}, \mathbf{a}, \mathbf{a}^{\prime}\right)$ and at the onset of gliogenesis ( $\left.36 \mathrm{hpf}, \mathbf{b}, \mathbf{b}^{\prime}\right)$ in wild-type (a,b) and sulf $2 a$ depleted (a',b') contexts. In 24 hpf wild-type embryos (a), the ventral-most progenitors of the $\mathrm{p} 3$ domain (green), adjacent to the medial floor plate (MFP, brown), express the highthreshold Shh responsive gene Nkx2.2a and generate V3 interneurons. Sulf2a (turquoise blue frame), expressed in dorsally located Olig2/pMN progenitors (red), limits the dorsal extent of high-threshold Shh response to prevent activation of $\mathrm{Nkx} 2.2 \mathrm{a}$ and thus allows maintenance of Olig2 expression (red) required to orient these cells toward the MN lineage. When sulf $2 a$ is depleted (a'), the range of high-threshold Shh response extends dorsally, leading to dorsal misexpression of $\mathrm{Nkx} 2.2 \mathrm{a}$ which in turn represses Olig2. Subsequent changes in the sizes of the $\mathrm{p} 3$ and $\mathrm{pMN}$ domains cause overproduction of V3 interneurons at the expense of MNs. In 36hpf wild-type embryos (b), the Shh source has expanded in the former Nkx2.2a/p3 domain to form the lateral floor plate (LFP, brown). Immediately adjacent progenitors, i.e. the ventral-most cells of the Olig $2 / \mathrm{pMN}$ domain, because they activate high-threshold Shh response, upregulate $\mathrm{Nkx} 2.2 \mathrm{a}$. At that stage, $\mathrm{Nkx} 2.2 \mathrm{a}$ no more represses Olig2, leading to formation of the $\mathrm{p}^{*}$ domain (yellow), populated by progenitors co-expressing Olig2 and $\mathrm{Nkx2.2a}$. Sulf2a expression (turquoise blue frame) is excluded from $\mathrm{p}^{\star}$ cells but maintained in dorsally located Olig2 progenitors (red) where the enzyme, again, prevents high-threshold Shh response. Then, the two distinct populations of Olig2 progenitors, expressing Nkx2.2a (yellow) or not (red) generate the myelinating (yellow) and non-myelinating (red) OPC, respectively. In $36 \mathrm{hpf}$ sulf $2 a$-depleted embryos (b'), LFP formation is not affected but, because of the dorsal expansion of the $\mathrm{Nkx} 2.2 \mathrm{a} / \mathrm{p} 3$ domain that occurred at earlier stage, the dorsally adjacent progenitors express Nkx2.2a but not Olig2 (green), in contrast to the wild-type situation. Nonetheless, Sulf2a depletion, again, allows activation of high-threshold Shh response that causes dorsal misexpression of Nkx2.2a. Subsequently, the $\mathrm{p}^{\star}$ domain (yellow), while positioned dorsally, forms properly but, the reduced size of the Olig2/pMN domain leads to a deficit in progenitors that only express Olig2 and, consequently to a strong reduction in the population of non-myelinating OPCs (red). It has to be noted that the population of myelinating OPC (yellow) is also mildly reduced possibly due to a secondary effect of aberrant neuronal populations (see discussion).

exposures. Ten $0.5 \mu \mathrm{m} z$ intervals were maximum $z$-projected and patched 2 fluorescent signal was measured in a rectangle of $9 \mu \mathrm{m}$ wide centred on the spinal cord lumen. To compare signal intensity profiles between different experimental conditions, data were normalised to the maximum value in each section analysed. Data for each embryo were collected from five trunk spinal cord sections and values represent the average of fluorescence intensity per section from five embryos for each experimental condition. Statistical analyses were performed using Prism5 software (GraphPad). Normality of data sets was tested using Kolmogorov-Smirnov's, D’Agostino and Pearson's and Shapiro-Wilk's tests using Prism5 (GraphPad). We considered datasets as normal when found normal in the three tests. Datasets following a normal distribution were compared with Student's $t$-test (unpaired two-tailed) while datasets that did not follow a normal distribution were compared using Mann Whitney's test (two-tailed). Patched 2 intensity profiles were analysed using two-way ANOVA (two-tailed) multiple comparison test. For each experiment, the statistical test used is indicated in figure legends. Statistical significance was accepted at a $\mathrm{p}<0.05$. All data are expressed as mean values per embryo \pm standard deviation (s.d).

Received: 21 August 2020; Accepted: 16 December 2020

Published online: 08 January 2021

\section{References}

1. Lamanna, W. C. et al. The heparanome-The enigma of encoding and decoding heparan sulfate sulfation. J. Biotechnol. 129, 290-307 (2007).

2. El Masri, R., Seffouh, A., Lortat-Jacob, H. \& Vivès, R. R. The, "in and out" of glucosamine 6-O-sulfation: The 6th sense of heparan sulfate. Glycoconj. J. 34, 285-298. https://doi.org/10.1007/s10719-016-9736-5 (2017).

3. Ai, X. et al. Substrate specificity and domain functions of extracellular heparan sulfate 6-O-endosulfatases, QSulf1 and QSulf2. J. Biol. Chem. 281, 4969-4976. https://doi.org/10.1074/jbc.M511902200 (2006).

4. Lamanna, W. C. et al. Heparan sulfate 6-O-endosulfatases: Discrete in vivo activities and functional co-operativity. Biochem. J. 400, 63-73. https://doi.org/10.1042/BJ20060848 (2006).

5. Seffouh, A. et al. HSulf sulfatases catalyze processive and oriented 6-O-desulfation of heparan sulfate that differentially regulates fibroblast growth factor activity. FASEB J. 27, 2431-2439. https://doi.org/10.1096/fj.12-226373 (2013).

6. Dhoot, G. K. et al. Regulation of Wnt signaling and embryo patterning by an extracellular sulfatase. Science 293, 1663-1666. https ://doi.org/10.1126/science.293.5535.1663 (2001).

7. Al Oustah, A. et al. Dynamics of sonic hedgehog signaling in the ventral spinal cord are controlled by intrinsic changes in source cells requiring sulfatase 1. Development 141, 1392-1403, https://doi.org/10.1242/dev.101717 (2014).

8. Danesin, C. et al. Ventral neural progenitors switch toward an oligodendroglial fate in response to increased sonic hedgehog (Shh) activity: Involvement of sulfatase 1 in modulating Shh signaling in the ventral spinal cord. J. Neurosci. 26, 5037-5048 (2006).

9. Touahri, Y. et al. Sulfatase 1 promotes the motor neuron-to-oligodendrocyte fate switch by activating Shh signaling in Olig2 progenitors of the embryonic ventral spinal cord. J. Neurosci. 32, 18018-18034. https://doi.org/10.1523/JNEUROSCI.3553-12.2012 (2012).

10. Ramsbottom, S. A., Maguire, R. J., Fellgett, S. W. \& Pownall, M. E. Sulf1 influences the Shh morphogen gradient during the dorsal ventral patterning of the neural tube in Xenopus tropicalis. Dev. Biol. 391, 207-218. https://doi.org/10.1016/j.ydbio.2014.04.010 (2014).

11. Jiang, W. et al. Sulfatase 2 modulates fate change from motor neurons to oligodendrocyte precursor cells through coordinated regulation of Shh signaling with sulfatase 1. Dev. Neurosci. 39, 361-374. https://doi.org/10.1159/000464284 (2017). 
12. Danesin, C. \& Soula, C. Moving the Shh source over time: What impact on neural cell diversification in the developing spinal cord? J. Dev. Biol. 5, https://doi.org/10.3390/jdb5020004 (2017).

13. Ribes, V. \& Briscoe, J. Establishing and interpreting graded Sonic Hedgehog signaling during vertebrate neural tube patterning: The role of negative feedback. Cold Spring Harb. Perspect. Biol. 1, a002014 (2009).

14. Dessaud, E., McMahon, A. P. \& Briscoe, J. Pattern formation in the vertebrate neural tube: A sonic hedgehog morphogen-regulated transcriptional network. Development 135, 2489-2503 (2008).

15. Dessaud, E. et al. Interpretation of the sonic hedgehog morphogen gradient by a temporal adaptation mechanism. Nature 450, 717-720 (2007).

16. Dessaud, E. et al. Dynamic assignment and maintenance of positional identity in the ventral neural tube by the morphogen sonic hedgehog. PLoS Biol 8, e1000382. https://doi.org/10.1371/journal.pbio.1000382 (2010).

17. Zhou, Q., Choi, G. \& Anderson, D. J. The bHLH transcription factor Olig2 promotes oligodendrocyte differentiation in collaboration with Nkx2.2. Neuron 31, 791-807 (2001).

18. Fu, H. et al. Dual origin of spinal oligodendrocyte progenitors and evidence for the cooperative role of Olig2 and Nkx2.2 in the control of oligodendrocyte differentiation. Development 129, 681-693 (2002).

19. Agius, E. et al. Converse control of oligodendrocyte and astrocyte lineage development by Sonic hedgehog in the chick spinal cord. Dev. Biol. 270, 308-321. https://doi.org/10.1016/j.ydbio.2004.02.015 (2004).

20. Sun, T. et al. Olig bHLH proteins interact with homeodomain proteins to regulate cell fate acquisition in progenitors of the ventral neural tube. Curr. Biol. 11, 1413-1420 (2001).

21. Ratzka, A. et al. Redundant function of the heparan sulfate 6-O-endosulfatases Sulf1 and Sulf2 during skeletal development. Dev. Dyn. 237, 339-353. https://doi.org/10.1002/dvdy.21423 (2008).

22. Ai, X. et al. SULF1 and SULF2 regulate heparan sulfate-mediated GDNF signaling for esophageal innervation. Development 134, 3327-3338. https://doi.org/10.1242/dev.007674 (2007).

23. Holst, C. R. et al. Secreted sulfatases Sulf1 and Sulf2 have overlapping yet essential roles in mouse neonatal survival. PLoS ONE 2, e575. https://doi.org/10.1371/journal.pone.0000575 (2007).

24. Nagamine, S., Koike, S., Keino-Masu, K. \& Masu, M. Expression of a heparan sulfate remodeling enzyme, heparan sulfate 6-O-endosulfatase sulfatase FP2, in the rat nervous system. Brain Res. Dev. Brain Res. 159, 135-143. https://doi.org/10.1016/j.devbrainre s.2005.07.006 (2005).

25. Ratzka, A., Mundlos, S. \& Vortkamp, A. Expression patterns of sulfatase genes in the developing mouse embryo. Dev. Dyn. 239, 1779-1788. https://doi.org/10.1002/dvdy.22294 (2010).

26. Ohayon, D. et al. Sulfatase 2 promotes generation of a spinal cord astrocyte subtype that stands out through the expression of Olig2. Glia 67, 1478-1495. https://doi.org/10.1002/glia.23621 (2019).

27. Gorsi, B., Whelan, S. \& Stringer, S. E. Dynamic expression patterns of 6-O endosulfatases during zebrafish development suggest a subfunctionalisation event for sulf2. Dev. Dyn. 239, 3312-3323. https://doi.org/10.1002/dvdy.22456 (2010).

28. Myers, P. Z., Eisen, J. S. \& Westerfield, M. Development and axonal outgrowth of identified motoneurons in the zebrafish. J. Neurosci 6, 2278-2289 (1986)

29. Schäfer, M., Kinzel, D. \& Winkler, C. Discontinuous organization and specification of the lateral floor plate in zebrafish. Dev. Biol. 301, 117-129. https://doi.org/10.1016/j.ydbio.2006.09.018 (2007).

30. Park, H. C., Shin, J. \& Appel, B. Spatial and temporal regulation of ventral spinal cord precursor specification by Hedgehog signaling. Development 131, 5959-5969. https://doi.org/10.1242/dev.01456 (2004).

31. Yang, L., Rastegar, S. \& Strähle, U. Regulatory interactions specifying Kolmer-Agduhr interneurons. Development 137, $2713-2722$. https://doi.org/10.1242/dev.048470 (2010).

32. Frese, M. A., Milz, F., Dick, M., Lamanna, W. C. \& Dierks, T. Characterization of the human sulfatase Sulf1 and its high affinity heparin/heparan sulfate interaction domain. J. Biol. Chem. 284, 28033-28044. https://doi.org/10.1074/jbc.M109.035808 (2009).

33. Tang, R. \& Rosen, S. D. Functional consequences of the subdomain organization of the sulfs. J. Biol. Chem. 284, 21505-21514. https://doi.org/10.1074/jbc.M109.028472 (2009).

34. Seffouh, A. et al. Expression and purification of recombinant extracellular sulfatase HSulf-2 allows deciphering of enzyme subdomain coordinated role for the binding and 6-O-desulfation of heparan sulfate. Cell Mol. Life Sci. 76, 1807-1819. https://doi. org/10.1007/s00018-019-03027-2 (2019).

35. Shin, J., Park, H. C., Topczewska, J. M., Mawdsley, D. J. \& Appel, B. Neural cell fate analysis in zebrafish using olig2 BAC transgenics. Methods Cell Sci. 25, 7-14. https://doi.org/10.1023/B:MICS.0000006847.09037.3a (2003).

36. Kucenas, S. et al. CNS-derived glia ensheath peripheral nerves and mediate motor root development. Nat. Neurosci. 11, $143-151$. https://doi.org/10.1038/nn2025 (2008).

37. Park, H. C., Mehta, A., Richardson, J. S. \& Appel, B. olig2 is required for zebrafish primary motor neuron and oligodendrocyte development. Dev. Biol. 248, 356-368 (2002).

38. Graham, V., Khudyakov, J., Ellis, P. \& Pevny, L. SOX2 functions to maintain neural progenitor identity. Neuron 39, 749-765. https ://doi.org/10.1016/s0896-6273(03)00497-5 (2003).

39. Bylund, M., Andersson, E., Novitch, B. G. \& Muhr, J. Vertebrate neurogenesis is counteracted by Sox1-3 activity. Nat. Neurosci. 6, 1162-1168. https://doi.org/10.1038/nn1131 (2003).

40. Kucenas, S., Snell, H. \& Appel, B. nkx2.2a promotes specification and differentiation of a myelinating subset of oligodendrocyte lineage cells in zebrafish. Neuron Glia Biol. 4, 71-81 (2008).

41. Ravanelli, A. M. et al. Sequential specification of oligodendrocyte lineage cells by distinct levels of Hedgehog and Notch signaling. Dev. Biol. 444, 93-106. https://doi.org/10.1016/j.ydbio.2018.10.004 (2018).

42. Barth, K. A. \& Wilson, S. W. Expression of zebrafish nk2.2 is influenced by sonic hedgehog/vertebrate hedgehog-1 and demarcates a zone of neuronal differentiation in the embryonic forebrain. Development 121, 1755-1768 (1995).

43. Guner, B. \& Karlstrom, R. O. Cloning of zebrafish nkx6.2 and a comprehensive analysis of the conserved transcriptional response to Hedgehog/Gli signaling in the zebrafish neural tube. Gene Expr. Patterns 7, 596-605, https://doi.org/10.1016/j.modgep.2007.01.002 (2007).

44. Shen, M. C. et al. Heat-shock-mediated conditional regulation of hedgehog/gli signaling in zebrafish. Dev. Dyn. 242, 539-549. https://doi.org/10.1002/dvdy.23955 (2013).

45. Schafer, M. et al. Hedgehog and retinoid signalling confines nkx2.2b expression to the lateral floor plate of the zebrafish trunk. Mech. Dev. 122, 43-56, https://doi.org/10.1016/j.mod.2004.09.002 (2005).

46. Ribes, V. et al. Distinct Sonic Hedgehog signaling dynamics specify floor plate and ventral neuronal progenitors in the vertebrate neural tube. Genes Dev. 24, 1186-1200. https://doi.org/10.1101/gad.559910 (2010).

47. Concordet, J. P. et al. Spatial regulation of a zebrafish patched homologue reflects the roles of sonic hedgehog and protein kinase A in neural tube and somite patterning. Development 122, 2835-2846 (1996).

48. Jacobs, C. T. \& Huang, P. Notch signalling maintains Hedgehog responsiveness via a Gli-dependent mechanism during spinal cord patterning in zebrafish. Elife 8, https://doi.org/10.7554/eLife.49252 (2019).

49. Novitch, B. G., Chen, A. I. \& Jessell, T. M. Coordinate regulation of motor neuron subtype identity and pan-neuronal properties by the bHLH repressor Olig2. Neuron 31, 773-789 (2001).

50. Ravanelli, A. M. \& Appel, B. Motor neurons and oligodendrocytes arise from distinct cell lineages by progenitor recruitment. Genes Dev. 29, 2504-2515. https://doi.org/10.1101/gad.271312.115 (2015). 
51. Baraban, M., Mensch, S. \& Lyons, D. A. Adaptive myelination from fish to man. Brain Res. 1641, 149-161. https://doi.org/10.1016/j. brainres.2015.10.026 (2016).

52. Li, F., Shi, W., Capurro, M. \& Filmus, J. Glypican-5 stimulates rhabdomyosarcoma cell proliferation by activating Hedgehog signaling. J. Cell Biol. 192, 691-704. https://doi.org/10.1083/jcb.201008087 (2011).

53. Witt, R. M. et al. Heparan sulfate proteoglycans containing a glypican 5 core and 2-O-sulfo-iduronic acid function as Sonic Hedgehog co-receptors to promote proliferation. J. Biol. Chem. 288, 26275-26288. https://doi.org/10.1074/jbc.M112.438937 (2013).

54. Capurro, M., Shi, W., Izumikawa, T., Kitagawa, H. \& Filmus, J. Processing by convertases is required for glypican-3-induced inhibition of Hedgehog signaling. J. Biol. Chem. 290, 7576-7585. https://doi.org/10.1074/jbc.M114.612705 (2015).

55. Capurro, M. et al. Glypican-6 promotes the growth of developing long bones by stimulating Hedgehog signaling. J. Cell Biol 216, 2911-2926. https://doi.org/10.1083/jcb.201605119 (2017).

56. Wojcinski, A., Nakato, H., Soula, C. \& Glise, B. DSulfatase-1 fine-tunes Hedgehog patterning activity through a novel regulatory feedback loop. Dev. Biol. 358, 168-180. https://doi.org/10.1016/j.ydbio.2011.07.027 (2011).

57. Kirby, B. B. et al. In vivo time-lapse imaging shows dynamic oligodendrocyte progenitor behavior during zebrafish development. Nat. Neurosci. 9, 1506-1511. https://doi.org/10.1038/nn1803 (2006).

58. Haeussler, M. et al. Evaluation of off-target and on-target scoring algorithms and integration into the guide RNA selection tool CRISPOR. Genome Biol. 17, 148. https://doi.org/10.1186/s13059-016-1012-2 (2016).

59. Kimmel, C. B., Ballard, W. W., Kimmel, S. R., Ullmann, B. \& Schilling, T. F. Stages of embryonic development of the zebrafish. Dev. Dyn. 203, 253-310. https://doi.org/10.1002/aja.1002030302 (1995).

60. Krauss, S., Concordet, J. P. \& Ingham, P. W. A functionally conserved homolog of the Drosophila segment polarity gene hh is expressed in tissues with polarizing activity in zebrafish embryos. Cell 75, 1431-1444 (1993).

61. Serluca, F. C. \& Fishman, M. C. Pre-pattern in the pronephric kidney field of zebrafish. Development 128, 2233-2241 (2001).

62. Xu, Q., Holder, N., Patient, R. \& Wilson, S. W. Spatially regulated expression of three receptor tyrosine kinase genes during gastrulation in the zebrafish. Development 120, 287-299 (1994).

63. Denkers, N., García-Villalba, P., Rodesch, C. K., Nielson, K. R. \& Mauch, T. J. FISHing for chick genes: Triple-label whole-mount fluorescence in situ hybridization detects simultaneous and overlapping gene expression in avian embryos. Dev. Dyn. 229, 651-657. https://doi.org/10.1002/dvdy.20005 (2004).

64. Andrieu, C. et al. MMP14 is required for delamination of chick neural crest cells independently of its catalytic activity. Development 147, https://doi.org/10.1242/dev.183954 (2020).

\section{Acknowledgements}

We are grateful to Developmental Studies Hybridoma Bank (Iowa City, IA, USA) for supplying monoclonal antibodies. We thank B. Appel, S. Temple, U. Strahle, C. Winkler, P. Blader, C. Houart and R. Karlstrom for sharing transgenic lines and reagents, E. Theveneau and B. Benazeraf for critical reading and help on the manuscript; A. Laire and the animal facility staff for zebrafish care and the Toulouse Regional Imaging Platform for technical assistance in confocal microscopy. Work in C.S.' lab was supported by grants from Agence Nationale de la Recherche (ANR-15-CE16-0014-02), Centre National de la Recherche Scientifique (CNRS), Fondation pour l'Aide à la Recherche sur la Sclérose en Plaques (ARSEP), Fondation ARC pour la Recherche sur le Cancer and Université de Toulouse.

\section{Author contributions}

C.D. planned the experiments. C.D., R.D.G., N.E., V.B., D.O. and B.G. performed and analysed experiments. P.C. participated in imaging the data. C.D. and C.S. supervised the project and wrote the manuscript. All authors read and approved the final manuscript.

\section{Competing interests}

The authors declare no competing interests.

\section{Additional information}

Supplementary Information The online version contains supplementary material available at https://doi. org/10.1038/s41598-020-80455-2.

Correspondence and requests for materials should be addressed to C.D.

Reprints and permissions information is available at www.nature.com/reprints.

Publisher's note Springer Nature remains neutral with regard to jurisdictional claims in published maps and institutional affiliations.

\footnotetext{
(c) (i) Open Access This article is licensed under a Creative Commons Attribution 4.0 International cc) License, which permits use, sharing, adaptation, distribution and reproduction in any medium or format, as long as you give appropriate credit to the original author(s) and the source, provide a link to the Creative Commons licence, and indicate if changes were made. The images or other third party material in this article are included in the article's Creative Commons licence, unless indicated otherwise in a credit line to the material. If material is not included in the article's Creative Commons licence and your intended use is not permitted by statutory regulation or exceeds the permitted use, you will need to obtain permission directly from the copyright holder. To view a copy of this licence, visit http://creativecommons.org/licenses/by/4.0/.
}

(C) The Author(s) 2021 